Article

\title{
Solution of Inhomogeneous Fractional Differential Equations with Polynomial Coefficients in Terms of the Green's Function, in Nonstandard Analysis
}

\author{
Tohru Morita ${ }^{1, *}$ and Ken-ichi Sato ${ }^{2}$ \\ 1 Graduate School of Information Sciences, Tohoku University, Sendai 980-8577, Japan \\ 2 Kurume Library on Mathematics, Koriyama 963-8846, Japan; kensatokurume@ybb.ne.jp \\ * Correspondence: senmm@jcom.home.ne.jp; Tel.: +81-22-278-6186
}

check for

updates

Citation: Morita, T.; Sato, K.-i.

Solution of Inhomogeneous

Fractional Differential Equations with

Polynomial Coefficients in Terms of

the Green's Function, in Nonstandard

Analysis. Mathematics 2021, 9, 1944.

https://doi.org/10.3390/math9161944

Academic Editor: Christopher

Goodrich

Received: 13 July 2021

Accepted: 10 August 2021

Published: 15 August 2021

Publisher's Note: MDPI stays neutral with regard to jurisdictional claims in published maps and institutional affiliations.

Copyright: (c) 2021 by the authors. Licensee MDPI, Basel, Switzerland. This article is an open access article distributed under the terms and conditions of the Creative Commons Attribution (CC BY) license (https:/ / creativecommons.org/licenses/by/ $4.0 /)$.

Abstract: Discussions are presented by Morita and Sato in Mathematics 2017; 5, 62: 1-24, on the problem of obtaining the particular solution of an inhomogeneous ordinary differential equation with polynomial coefficients in terms of the Green's function, in the framework of distribution theory. In the present paper, a compact recipe in nonstandard analysis is presented, which is applicable to an inhomogeneous ordinary and also fractional differential equation with polynomial coefficients. The recipe consists of three theorems, each of which provides the particular solution of a differential equation for an inhomogeneous term, satisfying one of three conditions. The detailed derivation of the applications of these theorems is given for a simple fractional differential equation and an ordinary differential equation.

Keywords: Green's function; fractional differential equations with polynomial coefficients; Kim and O's differential equation; nonstandard analysis; distribution theory; operational calculus

\section{Introduction}

We consider a fractional differential equation, which takes the following form:

$$
p_{n}\left(t,{ }_{R} D_{t}\right) u(t):=\sum_{l=0}^{n} a_{l}(t)_{R} D_{t}^{\rho_{l}} u(t)=f(t),
$$

where $n \in \mathbb{Z}_{>-1}, a_{l}(t)$ for $l \in \mathbb{Z}_{[0, n]}$ are polynomials of $t, \rho_{l} \in \mathbb{C}$ for $l \in \mathbb{Z}_{[0, n]}$ satisfy $\operatorname{Re} \rho_{0}>\operatorname{Re} \rho_{1} \geq \cdots \geq \operatorname{Re} \rho_{n}$ and $\operatorname{Re} \rho_{0}>0$. We use Heaviside's step function $H(t)$, which is equal to 1 if $t>0$ and, to 0 if $t \leq 0$. Here, ${ }_{R} D_{t}^{\rho_{l}}$ are the Riemann-Liouville fractional integrals and derivatives defined by the following definition; see $[1,2]$.

Remark 1. In solving Equation (1), we assume that $f(t)$ satisfies $f(t)=f(t) H(t)$, and we use the solution $u(t)=0$ for $t \leq 0$. As a result, the solution $u(t)$ satisfies $u(t)=u(t) H(t)$. In this notation, we use the following definition for $\tau=0$.

Definition 1. Let $t \in \mathbb{R}, \tau \in \mathbb{R}, u(t)$ satisfy $u(t)=u(t) H(t-\tau), \lambda \in \mathbb{C}_{+}, n \in \mathbb{Z}_{>-1}$ and $\rho=n-\lambda$. Then, ${ }_{R} D_{t}^{-\lambda} u(t)$ is the Riemann-Liouville fractional integral defined by the following:

$$
\begin{aligned}
{ }_{R} D_{t}^{-\lambda} u(t) & =\frac{1}{\Gamma(\lambda)} \int_{-\infty}^{t}(t-\xi)^{\lambda-1} u(\xi) H(\xi-\tau) d \xi \\
& =\frac{1}{\Gamma(\lambda)} \int_{\tau}^{t}(t-\xi)^{\lambda-1} u(\xi) d \xi, \quad t>\tau
\end{aligned}
$$


and ${ }_{R} D_{t}^{-\lambda} u(t)=0$ for $t \leq \tau$, where $\Gamma(\lambda)$ is the gamma function, and ${ }_{R} D_{t}^{\rho} u(t)={ }_{R} D_{t}^{n-\lambda} u(t)$ is the Riemann-Liouville fractional derivative defined by the following:

$$
{ }_{R} D_{t}^{\rho} u(t)={ }_{R} D_{t}^{n-\lambda} u(t)=\frac{d^{n}}{d t^{n}}\left[{ }_{R} D_{t}^{-\lambda} u(t)\right] \cdot H(t-\tau),
$$

when $n \geq \operatorname{Re} \lambda$, and ${ }_{R} D_{t}^{n} u(t)=\frac{d^{n}}{d t^{n}} u(t) \cdot H(t-\tau)$ when $\rho=n \in \mathbb{Z}_{>-1}$.

Here, $\mathbb{Z}, \mathbb{R}$ and $\mathbb{C}$ are the sets of all integers, all real numbers and all complex numbers, respectively, and $\mathbb{Z}_{>a}=\{n \in \mathbb{Z} \mid n>a\}, \mathbb{Z}_{<b}=\{n \in \mathbb{Z} \mid n<b\}$ and $\mathbb{Z}_{[a, b]}=\{n \in \mathbb{Z} \mid a \leq$ $n \leq b\}$ for $a, b \in \mathbb{Z}$ satisfying $a<b$. We also use $\mathbb{R}_{>a}=\{x \in \mathbb{R} \mid x>a\}$ for $a \in \mathbb{R}$, and $\mathbb{C}_{+}=\{z \in \mathbb{C} \mid \operatorname{Re} z>0\}$.

In accordance with Definition 1, we adopt the following:

$$
{ }_{R} D_{t}^{\rho} \frac{1}{\Gamma(v)} t^{v-1} H(t)= \begin{cases}\frac{1}{\Gamma(v-\rho)} t^{\nu-\rho-1} H(t), & v-\rho \in \mathbb{C} \backslash \mathbb{Z}_{<1}, \\ 0, & v-\rho \in \mathbb{Z}_{<1},\end{cases}
$$

for $v \in \mathbb{C} \backslash \mathbb{Z}_{<1}$. Here ${ }_{R} D_{t}$ is used in place of usually used notation ${ }_{0} D_{R}$, in order to show that the variable is $t$.

In [3,4], discussions are made of an ordinary differential equation, which is expressed by (1) for $\rho_{l}=n-l$, in terms of distribution theory, and with the aid of the analytic continuation of Laplace transform, respectively. In those papers, solutions are given of differential equations with an inhomogeneous term $f(t)$, which satisfies one of the following three conditions.

Condition 1. (i) $f(t)=f_{0}(t) H(t)$, where $f_{0}(t)$ multiplied by $H(t)$ is locally integrable on $\mathbb{R}$. (ii) $f(t)={ }_{R} D_{t}^{\beta}\left[f_{\beta}(t) H(t)\right]$, where $\beta \in \mathbb{C} \backslash \mathbb{Z}_{>-1}$, and $f_{\beta}(t)$ multiplied by $H(t)$ is locally integrable on $\mathbb{R}$.

(iii) $f(t)={ }_{R} D_{t}^{\beta+1} H(t)=\frac{1}{\Gamma(-\beta)} t^{-\beta-1} H(t)$, where $\beta \in \mathbb{C} \backslash \mathbb{Z}_{>-1}$.

\subsection{Recipe of Solution of Differential Equation, in Distribution Theory}

In a recent paper [5], the solution of Euler's differential equation in distribution theory is compared with the solution in nonstandard analysis. In distribution theory [6-8], we use distribution $\tilde{H}(t)$, which corresponds to function $H(t)$, differential operator $D$ and distribution $\delta(t)=D \tilde{H}(t)$, which is called Dirac's delta function.

Remark 2. In solving (1) in [3], the Green's function $\tilde{G}(t, \tau)$ in distribution theory is introduced by

$$
p_{n}(t, D) \tilde{G}(t, \tau)=\delta(t-\tau)
$$

Lemma 1. Let $u_{c}(t, \tau)$ be a complementary solution of Equation (1) for $t>\tau$, and $G_{0}(t, \tau)$, which is given by the following,

$$
G_{0}(t, \tau)=u_{c}(t, \tau) H(t-\tau)
$$

satisfy

$$
{ }_{R} D_{t}^{-1}\left[p_{n}\left(t,{ }_{R} D_{t}\right) G_{0}(t, \tau)\right]=H(t-\tau)= \begin{cases}1, & t>\tau, \\ 0, & t \leq \tau .\end{cases}
$$

Then, $\tilde{G}(t, \tau)=G_{0}(t, \tau) \tilde{H}(t-\tau)$ is the Green's function $\tilde{G}(t, \tau)$ defined in Remark 2. 
Lemma 2. In [3], it was shown that if $f(t)$ satisfies Condition $1(i)$ and $G_{0}(t, \tau)$ is the one given in Lemma 1, $u_{f}(t)$, given by the following,

$$
u_{f}(t)=\int_{0}^{t} G_{0}(t, \tau) f(\tau) d \tau
$$

is a particular solution of Equation (1).

A proof of this lemma is given to show that if $G_{0}(t, \tau)$ satisfies (7), $u_{f}(t)$ given by (8) is a particular solution of (1), in Section 1.4.

\subsection{Preliminaries on Nonstandard Analysis}

In the present paper, we use nonstandard analysis [9], where infinitesimal numbers are used. We denote the set of all infinitesimal real numbers by $\mathbb{R}^{0}$. We also use $\mathbb{R}_{>0}^{0}=$ $\left\{\epsilon \in \mathbb{R}^{0} \mid \epsilon>0\right\}$, which is such that if $\epsilon \in \mathbb{R}_{>0}^{0}$ and $N \in \mathbb{Z}_{>0}$, then $\epsilon<\frac{1}{N}$. We use $\mathbb{R}^{n s}$, which has subsets $\mathbb{R}$ and $\mathbb{R}^{0}$. If $x \in \mathbb{R}^{n s}$ and $x \notin \mathbb{R}, x$ is expressed as $x_{1}+\epsilon$ by $x_{1} \in \mathbb{R}$ and $\epsilon \in \mathbb{R}^{0}$, where $x_{1}$ may be $0 \in \mathbb{R}$. Equation $x \simeq y$ for $x \in \mathbb{R}^{n s}$ and $y \in \mathbb{R}^{n s}$, is used, when $x-y \in \mathbb{R}^{0}$. We denote the set of all infinitesimal complex numbers by $\mathbb{C}^{0}$, which is the set of complex numbers $z$, which satisfy $|\operatorname{Re} z|+|\operatorname{Im} z| \in \mathbb{R}^{0}$. We use $\mathbb{C}^{n s}$, which has subsets $\mathbb{C}$ and $\mathbb{C}^{0}$. If $z \in \mathbb{C}^{n s}$ and $z \notin \mathbb{C}, z$ is expressed as $z_{1}+\epsilon$ by $z_{1} \in \mathbb{C}$ and $\epsilon \in \mathbb{C}^{0}$, where $z_{1}$ may be $0 \in \mathbb{C}$.

Remark 3. In nonstandard analysis [9], in addition to infinitesimal numbers, we use unlimited numbers, which are often called infinite numbers. In the present paper, we do not use them, but if we use them, we have to consider sets $\mathbb{R}^{\infty}$ and $\mathbb{C}^{\infty}$ such that if $\omega \in \mathbb{R}^{\infty}$, there exists $\in \in \mathbb{R}^{0}$ satisfying $\omega=\frac{1}{\epsilon}$, and if $\omega \in \mathbb{C}^{\infty}$, there exists $\in \in \mathbb{C}^{0}$ satisfying $\omega=\frac{1}{\epsilon}$, and to include these sets as subsets of $\mathbb{R}^{n s}$ and $\mathbb{C}^{n s}$, respectively.

In place of (4), we now use the following:

$$
{ }_{R} D_{t}^{\rho} \frac{1}{\Gamma(v+\epsilon)} t^{\nu-1+\epsilon} H(t)=\frac{1}{\Gamma(v-\rho+\epsilon)} t^{\nu-\rho-1+\epsilon} H(t),
$$

for all $\rho \in \mathbb{C}$ and $\nu \in \mathbb{C}$.

Remark 4. When $\epsilon \in \mathbb{R}^{0}$ or $\epsilon \in \mathbb{C}^{0}$, we often ignore terms of $O(\epsilon)$ compared with a term of $O\left(\epsilon^{0}\right)$. For instance, when $v \in \mathbb{R}_{>0}$ and $v-\rho \in \mathbb{R}_{>0}$, we adopt $\frac{1}{\Gamma(v+\epsilon)} t^{v-1+\epsilon} H(t) \simeq \frac{1}{\Gamma(v)} t^{\nu-1+\epsilon} H(t)$, and also

$$
{ }_{R} D_{t}^{\rho} \frac{1}{\Gamma(v)} t^{v-1+\epsilon} H(t) \simeq \frac{1}{\Gamma(v-\rho)} t^{v-\rho-1+\epsilon} H(t),
$$

in place of (9). In the following, we often use " =" in place of " $\simeq "$.

In the study in nonstandard analysis, $\epsilon \in \mathbb{R}_{>0}^{0}$ is used, and $H(t)$ and $\delta(t)=D \tilde{H}(t)$, respectively, are replaced by $H_{\epsilon}(t):={ }_{R} D_{t}^{-\epsilon} H(t)=\frac{1}{\Gamma(\epsilon+1)} t^{\epsilon} H(t) \simeq t^{\epsilon} H(t)$ which tends to $H(t)$ in the limit $\epsilon \rightarrow 0$, and by the following:

$$
\delta_{\epsilon}(t):=\frac{d}{d t} H_{\epsilon}(t)={ }_{R} D_{t}^{1-\epsilon} H(t)=\epsilon t^{-1} H_{\epsilon}(t)=\frac{1}{\Gamma(\epsilon)} t^{\epsilon-1} H(t) \simeq \epsilon t^{\epsilon-1} H(t) .
$$


Lemma 3. Let $f(t-\tau)$, for $t \in \mathbb{R}_{>0}$ and $\tau \in \mathbb{R}$ satisfying $\tau \in[0, t]$, be expressed as $f(t-\tau)=$ $f(t)-f^{\prime}(t) \tau+O\left(\tau^{2}\right), \epsilon \in \mathbb{R}_{>0}^{0}$ and $\epsilon_{1} \in(0, \epsilon)$. Then, we have the following:

$$
\begin{aligned}
\int_{-\infty}^{\infty} \delta_{\epsilon_{1}}(t-\tau) f(\tau) H(\tau) d \tau & =\int_{0}^{t} \delta_{\epsilon_{1}}(\tau) f(t-\tau) d \tau=f(t) t^{\epsilon_{1}} H(t)+O\left(\epsilon_{1}\right) \\
& =f(t) H(t),
\end{aligned}
$$

in the limit $\epsilon_{1} \rightarrow 0$, and

$$
\int_{-\infty}^{\infty} \delta_{\epsilon}(t-\tau) f(\tau) H(\tau) d \tau=\int_{0}^{t} \delta_{\epsilon}(\tau) f(t-\tau) d \tau={ }_{R} D_{t}^{-\epsilon}[f(t) H(t)] .
$$

Proof. Equation (12) in this lemma is proved by using the following lemma. Since (11) shows that $\delta_{\epsilon}(t-\tau)={ }_{R} D_{t}^{1-\epsilon+\epsilon_{1}-\epsilon_{1}} H(t-\tau)={ }_{R} D_{t}^{-\epsilon+\epsilon_{1}} \delta_{\epsilon_{1}}(t-\tau)$, by applying ${ }_{R} D_{t}^{-\epsilon+\epsilon_{1}}$ to (12) and then taking the limit $\epsilon_{1} \rightarrow 0$, we obtain (13).

Lemma 4. Let $\rho=0$ or $\rho \in \mathbb{R}_{>0}$, and $\epsilon_{1} \in \mathbb{R}_{>0}^{0}$. Then

$$
\int_{0}^{t} \tau^{\rho} \delta_{\epsilon_{1}}(\tau) d \tau=\frac{1}{\Gamma\left(\epsilon_{1}\right)} \int_{0}^{t} \tau^{\rho} \tau^{\epsilon_{1}-1} d \tau=\frac{\epsilon_{1}}{\Gamma\left(1+\epsilon_{1}\right)\left(\rho+\epsilon_{1}\right)} t^{\rho+\epsilon_{1}} H(t),
$$

which is $\frac{1}{\Gamma\left(1+\epsilon_{1}\right)} t^{\epsilon_{1}}=t^{\epsilon_{1}}$ when $\rho=0$, and is $O\left(\epsilon_{1}\right)$ when $\rho \in \mathbb{R}_{>0}$.

\subsection{Summary of Sections $2-6$}

In Section 2, we give recipe of solution of a differential equation, in nonstandard analysis, where the differential equation is the following:

$$
p_{n}\left(t,{ }_{R} D_{t}\right) u(t)=\tilde{f}(t),
$$

and the inhomogeneous term $\tilde{f}(t)$ satisfies one of the following three conditions.

Condition 2. Let $\epsilon \in \mathbb{R}_{>0}^{0}$.

(i) $\tilde{f}(t)=f(t) H(t)$, where $f(t)$ multiplied by $H(t)$ is locally integrable on $\mathbb{R}$.

(ii) $\tilde{f}(t)={ }_{R} D_{t}^{\beta} \tilde{f}_{\beta}(t)$, where $\beta \in \mathbb{C}, \tilde{f}_{\beta}(t)={ }_{R} D_{t}^{-\epsilon}\left[f_{\beta}(t) H(t)\right]$, and $f_{\beta}(t)$ multiplied by $H(t)$ is locally integrable on $\mathbb{R}$.

(iii) $\tilde{f}(t)={ }_{R} D_{t}^{\beta} \tilde{f}_{\beta}(t)$, where $\beta \in \mathbb{C}$, and $\tilde{f}_{\beta}(t)={ }_{R} D_{t} H_{\epsilon}(t)=\delta_{\epsilon}(t)$.

A complementary solution of Equation (1) is also that of Equation (15).

In [10], an ordinary differential equation is expressed in terms of blocks of classified terms. When the equation is expressed by two blocks of classified terms, the complementary solutions are obtained by using Frobenius' method. In the present case, a block of classified terms $D^{\rho} u(t)$ for $\rho \in \mathbb{C}$ and takes the following form:

$$
D^{\rho} u(t)=\sum_{k=0}^{k_{x}} a_{k} t^{k \mu}{ }_{R} D_{t}^{\rho+k \mu} u(t)
$$

where $\mu \in \mathbb{C}_{+}$and $k_{x} \in \mathbb{Z}_{>-1}$. An equation which consists of two blocks of classified terms, is expressed by the following:

$$
D^{\rho} u(t)+D^{\rho-l \mu} u(t)=f(t),
$$

where $\rho \in \mathbb{C}_{+}$and $l \in \mathbb{Z}_{>0}$.

In [11,12], discussions are made of fractional differential equations with constant coefficients, where the differential equation for the Green's function is solved either by an 
operational calculus giving the Neumann series, or by changing it to an integral equation which is solved by iterations, following the discussion for the ordinary differential equation given in the book [13]. Kim and O [14] present the corresponding argument for a fractional differential equation of the form, where an example of a simple equation for which the Green's function was given.

It is the primary purpose of this paper to present the recipe in Section 2 and to give a derivation of full expressions of the Green's functions of a fractional differential equation:

$$
{ }_{R} D_{t}^{3 / 2} \tilde{u}(t)-a(t+b) \cdot{ }_{R} D_{t}^{1 / 2} \tilde{u}(t)=\tilde{f}(t),
$$

and of an associated ordinary differential equation:

$$
\frac{d}{d t} y(t)-a(t+b) \cdot y(t)=\tilde{f}(t)
$$

where $a \in \mathbb{C}$ and $b \in \mathbb{C}$ are constants. These studies are given in Sections 2.2 and 3-3.4, respectively. In Section 3.4, the operational calculus or the method of iterations is used.

Equations (18) and (19) for $b=0$ are expressed by the following:

$$
\begin{aligned}
p_{F}\left(t,{ }_{R} D_{t}\right) u(t) & :={ }_{R} D_{t}^{3 / 2} \tilde{u}(t)-a t \cdot{ }_{R} D_{t}^{1 / 2} \tilde{u}(t)=\tilde{f}(t), \\
p_{L}\left(t, \frac{d}{d t}\right) y(t) & :=\frac{d}{d t} y(t)-a t \cdot y(t)=\tilde{f}(t),
\end{aligned}
$$

which take the form (17) with $\mu=1, \rho=\frac{3}{2}$ and $l=2$, and with $\mu=1, \rho=1$ and $l=2$, respectively. The equation, which Kim and O treated in [14], is Equation (20) for $a=-1$. The particular solutions of Equations (20) and (21) with the aid of Theorems 2 and 3, are presented in Sections 5-5.5 and 5.7.

In Section 4.1, we consider the following differential equation satisfying Condition 2 (iii):

$$
{ }_{R} D_{t}^{\rho} u(t)-a t^{\lambda} \cdot{ }_{R} D_{t}^{\lambda-\alpha+\rho} u(t)={ }_{R} D_{t}^{\beta+1} H_{\epsilon}(t)=\frac{t^{-\beta-1+\epsilon}}{\Gamma(-\beta+\epsilon)} H(t),
$$

where $\rho \in \mathbb{C}_{+}, \lambda \in \mathbb{R}_{>0}, \alpha \in \mathbb{R}_{>0}$ and $\beta \in \mathbb{C}$. This equation takes the form (17) and its solution is derived by the operational calculus or the method of iterations.

Equations (20) and (21), satisfying Condition 2 (iii), are (22) for $\rho=\frac{3}{2}, \lambda=1$ and $\alpha=2$, and for $\rho=1, \lambda=1$ and $\alpha=2$, respectively. Their solutions, with the aid of iterations, are obtained from those of Equation (22) in Sections 4.2 and 4.3. Their solutions, with the aid of Theorems 1 and 3, are given in Sections 3.5 and 5.7, respectively. The solutions, by using Frobenius' method, are given in Section 5.6. In Section 5.7, nonstandard solutions, which involve infinitesimal terms, are presented.

Section 6 presents the conclusions.

\subsection{Proof of Lemma 2}

We write (7) as $\int_{\tau}^{t}\left[p_{n}\left(x,{ }_{R} D_{x}\right) G_{0}(x, \tau)\right] d x=H(t-\tau)$, and then by using (8), we have the following:

$$
\begin{aligned}
& \int_{0}^{t}\left[\int_{\tau}^{t} p_{n}\left(x,{ }_{R} D_{x}\right) G_{0}(x, \tau) d x\right] f(\tau) H(\tau) d \tau \\
& =\int_{0}^{t} d x\left[\int_{0}^{x} p_{n}\left(x,{ }_{R} D_{x}\right) G_{0}(x, \tau) f(\tau) H(\tau) d \tau\right]=\int_{0}^{t} p_{n}\left(x,{ }_{R} D_{x}\right) u_{f}(x) d x \\
& =\int_{0}^{t} H(t-\tau) f(\tau) H(\tau) d \tau=\int_{0}^{t} f(\tau) H(\tau) d \tau .
\end{aligned}
$$

By taking the derivatives of the third and the last member in this equation with respect to $t$, we confirm that (1) is satisfied by $u(t)=u_{f}(t)$. 


\section{Recipe of Solution of Differential Equation, in Nonstandard Analysis}

2.1. Solution of Equation (15) When Condition 2 (i) Is Satisfied

In obtaining a particular solution of Equation (15) for $\tilde{f}(t)$ satisfying Condition $2(\mathrm{i})$, in place of the Green's function defined in Remark 2, we use it as defined in the following definition.

Definition 2. For $\epsilon_{1} \in \mathbb{R}_{>0}^{0}$, the Green's function $G_{\epsilon_{1}}(t, \tau)$ of Equation (15) satisfies the following:

$$
\begin{aligned}
p_{n}\left(t,{ }_{R} D_{t}\right) G_{\epsilon_{1}}(t, \tau) & =\delta_{\epsilon_{1}}(t-\tau), \\
{ }_{R} D_{t}^{-1}\left[p_{n}\left(t,{ }_{R} D_{t}\right) G_{\epsilon_{1}}(t, \tau)\right] & =H_{\epsilon_{1}}(t-\tau) .
\end{aligned}
$$

Lemma 5. Let $G_{\epsilon_{1}}(t, \tau)$ be defined as in Definition 2, and $G_{0}(t, \tau)$ be the solution of Equation (7). Then, in the limit $\epsilon_{1} \rightarrow 0, G_{\epsilon_{1}}(t, \tau)$ tends to $G_{0}(t, \tau)$. In this situation, we call $G_{0}(t, \tau)$ the solution of Equation (25) in the limit $\epsilon_{1} \rightarrow 0$.

Proof. We conclude this since in the limit $\epsilon_{1} \rightarrow 0, \delta_{\epsilon_{1}}(t-\tau)$ and $H_{\epsilon_{1}}(t-\tau)$ in Equations (24) and (25) tend to 0 and 1 , respectively, at $t>\tau$.

In Sections 3.2-3.5, we use the following theorem.

Theorem 1. Let Condition 2 (i) be satisfied and $G_{\epsilon_{1}}(t, \tau)$ and $G_{0}(t, \tau)$ be given as in Lemma 5 . Then $u_{f}(t)$, which is given by the following:

$$
u_{f}(t)=\int_{0}^{t} G_{\epsilon_{1}}(t, \tau) \tilde{f}(\tau) d \tau=\int_{0}^{t} G_{0}(t, \tau) \tilde{f}(\tau) d \tau,
$$

in the limit $\epsilon_{1} \rightarrow 0$, is a particular solution of Equation (15) for the term $\tilde{f}(t)$.

Proof. By using Equations (26), (24) and (12), in this order, we obtain the following:

$$
\begin{aligned}
p_{n}\left(t,{ }_{R} D_{t}\right) u_{f}(t) & =p_{n}\left(t,{ }_{R} D_{t}\right) \int_{0}^{t} G_{\epsilon_{1}}(t, \tau) \tilde{f}(\tau) d \tau=\int_{0}^{t} \delta_{\epsilon_{1}}(t-\tau) \tilde{f}(\tau) d \tau \\
& =\tilde{f}(t)
\end{aligned}
$$

in the limit $\epsilon_{1} \rightarrow 0$.

2.2. Solution of Equation (15) When Condition 2 (ii) or (iii) Is Satisfied

Definition 3. When Condition 2 (ii) or (iii) is satisfied, we introduce a transformed differential equation of Equation (15), by the following:

$$
\tilde{p}_{n, \beta}\left(t,{ }_{R} D_{t}\right) w(t)=\tilde{f}_{\beta}(t),
$$

where $w(t)={ }_{R} D_{t}^{-\beta} u(t)$, and

$$
\tilde{p}_{n, \beta}\left(t,{ }_{R} D_{t}\right):={ }_{R} D_{t}^{-\beta} p_{n}\left(t,{ }_{R} D_{t}\right)_{R} D_{t}^{\beta} .
$$

Lemma 6. Let Condition 2 (ii) or (iii) be satisfied. Then, by Definition 3, when (28) holds, Equation (15) for $u(t)={ }_{R} D_{t}^{\beta} w(t)$ holds.

Definition 4. For $\in \in \mathbb{R}_{>0}^{0}$, the Green's function $G_{\beta, \epsilon}(t, \tau)$ of Equation (28) satisfies the following:

$$
\begin{aligned}
\tilde{p}_{n, \beta}\left(t,{ }_{R} D_{t}\right) G_{\beta, \epsilon}(t, \tau) & =\delta_{\epsilon}(t-\tau), \\
{ }_{R} D_{t}^{-1}\left[\tilde{p}_{n, \beta}\left(t,{ }_{R} D_{t}\right) G_{\beta, \epsilon}(t, \tau)\right] & =H_{\epsilon}(t-\tau) .
\end{aligned}
$$


Lemma 7. Let $G_{\beta, \epsilon}(t, \tau)$ be defined by Definition 4. Then, a particular solution of Equation (28) for the term $\tilde{f}_{\beta}(t)$ is given by the following:

$$
w_{f}(t)=\int_{0}^{t} G_{\beta, \epsilon}(t, \tau) f_{\beta}(\tau) H(\tau) d \tau,
$$

if Condition 2 (ii) is satisfied, and by $w_{f}(t)=G_{\beta, \epsilon}(t, 0)$, if Condition 2 (iii) is satisfied.

Proof. When Condition 2 (ii) is satisfied, by using Equations (32), (30) and (13), we obtain the following:

$$
\begin{aligned}
p_{n, \beta}\left(t,{ }_{R} D_{t}\right) w_{f}(t) & =p_{n, \beta}\left(t,{ }_{R} D_{t}\right) \int_{0}^{t} G_{\beta, \epsilon}(t, \tau) f_{\beta}(\tau) H(\tau) d \tau \\
& =\int_{0}^{t} \delta_{\epsilon}(t-\tau) f_{\beta}(\tau) H(\tau) d \tau={ }_{R} D_{t}^{-\epsilon}\left[f_{\beta}(t) H(t)\right] .
\end{aligned}
$$

When Condition 2 (iii) is satisfied, $\tilde{f}_{\beta}(t)=\delta_{\epsilon}(t)$; hence, (30) shows that $w_{f}(t)=G_{\beta, \epsilon}(t, 0)$ is a particular solution of $(28)$.

Definition 5. When Condition 2 (ii) or (iii) is satisfied, we introduce a transformed differential equation of Equation (28), by the following:

$$
\tilde{p}_{n, \beta-\epsilon+\epsilon_{1}}\left(t,{ }_{R} D_{t}\right) w_{\epsilon, \epsilon_{1}}(t)={ }_{R} D_{t}^{-\epsilon_{1}}\left[f_{\beta}(t) H(t)\right],
$$

where $\epsilon_{1} \in(0, \epsilon)$, and

$$
\begin{aligned}
\tilde{p}_{n, \beta-\epsilon+\epsilon_{1}}\left(t,{ }_{R} D_{t}\right) & :={ }_{R} D_{t}^{\epsilon-\epsilon_{1}} \tilde{p}_{n, \beta}\left(t,{ }_{R} D_{t}\right)_{R} D_{t}^{-\epsilon+\epsilon_{1}} \\
& ={ }_{R} D_{t}^{-\beta+\epsilon-\epsilon_{1}} p_{n}\left(t,{ }_{R} D_{t}\right)_{R} D_{t}^{\beta-\epsilon+\epsilon_{1}} .
\end{aligned}
$$

Lemma 8. Let Condition 2 (ii) or (iii) be satisfied. Then, by Definition 5, when (34) holds, Equation (28) for $w(t)={ }_{R} D_{t}^{-\epsilon+\epsilon_{1}} w_{\epsilon, \epsilon_{1}}(t)$ holds.

Lemma 9. Let $\tilde{p}_{n, \beta-\epsilon+\epsilon_{1}}\left(t,{ }_{R} D_{t}\right)$ be defined by (35), $G_{\beta, \epsilon}(t, \tau)$ be defined in Definition 4, and $G_{\beta, \epsilon, \epsilon_{1}}(t, \tau):={ }_{R} D_{t}^{\epsilon-\epsilon_{1}} G_{\beta, \epsilon}(t, \tau)$. Then $G_{\beta, \epsilon, \epsilon_{1}}(t, \tau)$ satisfies the following:

$$
\begin{aligned}
\tilde{p}_{n, \beta-\epsilon+\epsilon_{1}}\left(t,{ }_{R} D_{t}\right) G_{\beta, \epsilon, \epsilon_{1}}(t, \tau) & =\delta_{\epsilon_{1}}(t-\tau), \\
{ }_{R} D_{t}^{-1}\left[\tilde{p}_{n, \beta-\epsilon+\epsilon_{1}}\left(t,{ }_{R} D_{t}\right) G_{\beta, \epsilon, \epsilon_{1}}(t, \tau)\right] & =H_{\epsilon_{1}}(t-\tau) .
\end{aligned}
$$

These equations show that when $G_{\beta, \epsilon, \epsilon_{1}}(t, \tau)$ exists, its limit $G_{\beta, \epsilon, 0}(t, \tau)$ as $\epsilon_{1} \rightarrow 0$ is expressed by the following:

$$
G_{\beta, \epsilon, 0}(t, \tau)=w_{\epsilon, c}(t) H(t-\tau),
$$

in terms of a complementary function $w_{\epsilon, c}(t)$ of Equation (34) for $\epsilon-\epsilon_{1}=\epsilon$.

Remark 5. Lemma 9 shows that we have the following relations:

$$
G_{\beta, \epsilon}(t, \tau)={ }_{R} D_{t}^{-\epsilon+\epsilon_{1}} G_{\beta, \epsilon, \epsilon_{1}}(t, \tau)={ }_{R} D_{t}^{-\epsilon} G_{\beta, \epsilon, 0}(t, \tau) .
$$

By using these relations, we can obtain any one of $G_{\beta, \epsilon}(t, \tau), G_{\beta, \epsilon, \epsilon_{1}}(t, \tau)$ and $G_{\beta, \epsilon, 0}(t, \tau)$, from any other. 
Lemma 10. Let Condition 2 (ii) be satisfied, and $G_{\beta, \epsilon, \epsilon_{1}}(t, \tau)$ be defined in Lemma 9. Then a particular solution of Equation (34) for the term ${ }_{R} D_{t}^{-\epsilon_{1}}\left[f_{\beta}(t) H(t)\right]$ is given by the following:

$$
w_{\epsilon, \epsilon_{1}}(t)=\int_{0}^{t} G_{\beta, \epsilon, \epsilon_{1}}(t, \tau) f_{\beta}(\tau) H(\tau) d \tau
$$

Lemma 8 shows that in this case, $w_{f}(t)$ given by $w_{f}(t)={ }_{R} D_{t}^{-\epsilon+\epsilon_{1}} w_{\epsilon, \epsilon_{1}}(t)$ is a particular solution of Equation (28) for the term $\tilde{f}_{\beta}(t)$.

Proof. By using Equations (40), (36) and (13), we obtain the following:

$$
\begin{aligned}
& \tilde{p}_{n, \beta-\epsilon+\epsilon_{1}}\left(t,{ }_{R} D_{t}\right) w_{\epsilon, \epsilon_{1}}(t)=\tilde{p}_{n, \beta-\epsilon+\epsilon_{1}}\left(t,{ }_{R} D_{t}\right) \int_{0}^{t} G_{\beta, \epsilon, \epsilon_{1}}(t, \tau) f_{\beta}(\tau) H(\tau) d \tau \\
& =\int_{0}^{t} \delta_{\epsilon_{1}}(t-\tau) f_{\beta}(\tau) H(\tau) d \tau={ }_{R} D_{t}^{-\epsilon_{1}}\left[f_{\beta}(t) H(t)\right] .
\end{aligned}
$$

By using Lemmas 6, 7 and 10, we confirm the following theorem.

Theorem 2. Let Condition 2 (ii) be satisfied, $G_{\beta, \epsilon}(t, \tau)$ satisfy Equations (30) and (31), $G_{\beta, \epsilon, \epsilon_{1}}(t, \tau)$ satisfy Equations (36) and (37), $G_{\beta, \epsilon, 0}(t, \tau)$, which is the limit of $G_{\beta, \epsilon, \epsilon_{1}}(t, \tau)$ as $\epsilon_{1} \rightarrow 0$, be expressed by (38), as stated in Lemma 9 , and $w_{f}(t)$ and $u_{f}(t)$ be given by the following:

$$
\begin{aligned}
w_{f}(t) & :=\int_{0}^{t} G_{\beta, \epsilon}(t, \tau) f_{\beta}(\tau) d \tau={ }_{R} D_{t}^{-\epsilon+\epsilon_{1}} \int_{0}^{t} G_{\beta, \epsilon, \epsilon_{1}}(t, \tau) f_{\beta}(\tau) d \tau \\
& ={ }_{R} D_{t}^{-\epsilon} \int_{0}^{t} G_{\beta, \epsilon, 0}(t, \tau) f_{\beta}(\tau) d \tau
\end{aligned}
$$

and $u_{f}(t):={ }_{R} D_{t}^{\beta} w_{f}(t)$. Then, $w_{f}(t)$ and $u_{f}(t)$ are particular solutions of Equations (28) and (15), respectively.

By using Lemmas 6, 7 and 9, we confirm the following theorem.

Theorem 3. Let Condition 2 (iii) be satisfied, and $G_{\beta, \epsilon}(t, 0), G_{\beta, \epsilon, \epsilon_{1}}(t, 0)$ and $G_{\beta, \epsilon, 0}(t, 0)$ be defined as in Theorem 2, and $w_{f}(t)$ be given by the following:

$$
w_{f}(t):=G_{\beta, \epsilon}(t, 0)={ }_{R} D_{t}^{-\epsilon+\epsilon_{1}} G_{\beta, \epsilon, \epsilon}(t, 0)={ }_{R} D_{t}^{-\epsilon} G_{\beta, \epsilon, 0}(t, 0),
$$

and $u_{f}(t)={ }_{R} D_{t}^{\beta} w_{f}(t)$.

Then, $G_{\beta, \epsilon, 0}(t, 0)$ has an expression given by $(38)$, and $w_{f}(t)$ and $u_{f}(t)$ are particular solutions of Equations (28) and (15), respectively.

\section{Solution of Equations (18) and (19) by Theorem 1}

We give solutions of fractional differential Equation (18) and ordinary differential Equation (19).

3.1. Complementary Solutions of Equations (18) and (19)

Lemma 11. The complementary solution of Equation (19) is $y(t)=C y_{1}(t)$, where the following holds:

$$
y_{1}(t)=e^{\frac{1}{2} a\left(t^{2}+2 b t\right)}=\sum_{k=0}^{\infty} \frac{a^{k}}{2^{k}} \sum_{l=0}^{k} \frac{2^{k-l} b^{k-l}}{(k-l) ! l !} t^{k+l}, \quad t>0
$$


and $C$ is a constant. When $b=0$, this becomes the corresponding solution of Equation (21) given by the following:

$$
y_{1}(t)=e^{\frac{1}{2} a t^{2}}=\sum_{k=0}^{\infty} \frac{a^{k}}{k ! 2^{k}} t^{2 k}, \quad t>0
$$

Proof. We see the following holds:

$$
y_{1}(t)=e^{\frac{1}{2} a\left(t^{2}+2 b t\right)}=\sum_{k=0}^{\infty} \frac{a^{k}}{k ! 2^{k}} t^{k}(t+2 b)^{k}=\sum_{k=0}^{\infty} \frac{a^{k}}{k ! 2^{k}} t^{k} \sum_{l=0}^{k} \frac{k ! 2^{k-l} b^{k-l}}{(k-l) ! l !} t^{l},
$$

which gives (44).

With the aid of (44) and (45), we obtain the following lemma.

Lemma 12. The complementary solution of (18) is given by $u(t)=C u_{1}(t)$, where the following holds:

$$
u_{1}(t)={ }_{R} D_{t}^{-1 / 2} y_{1}(t)=\sum_{k=0}^{\infty} \frac{a^{k}}{2^{k}} \sum_{l=0}^{k} \frac{2^{k-l} b^{k-l}}{(k-l) ! l !} \cdot \frac{\Gamma(k+l+1)}{\Gamma\left(k+l+\frac{3}{2}\right)} t^{k+l+1 / 2}, \quad t>0 .
$$

When $b=0$, this becomes the corresponding solution of Equation (20) given by the following:

$$
u_{1}(t)={ }_{R} D_{t}^{-1 / 2} y_{1}(t)=\frac{t^{1 / 2}}{\Gamma\left(\frac{3}{2}\right)} \cdot{ }_{2} F_{2}\left(1, \frac{1}{2} ; \frac{3}{4}, \frac{5}{4} ; \frac{1}{2} a t^{2}\right), \quad t>0,
$$

where ${ }_{2} F_{2}\left(\alpha_{1}, \alpha_{2} ; \beta_{1}, \beta_{2} ; z\right)=\sum_{k=0}^{\infty} \frac{\left(\alpha_{1}\right)_{k}\left(\alpha_{2}\right)_{k}}{k !\left(\beta_{1}\right)_{k}\left(\beta_{2}\right)_{k}} z^{k},(\alpha)_{k}=\prod_{l=0}^{k-1}(\alpha+l)$ if $k \in \mathbb{Z}_{>0}$, and $(\alpha)_{k}=1$ if $k=0$.

Proof. When $b=0$, by using (45), we obtain the following:

$$
u_{1}(t)={ }_{R} D_{t}^{-1 / 2} y_{1}(t)=\sum_{k=0}^{\infty} \frac{a^{k} \Gamma(2 k+1)}{k ! 2^{k} \Gamma\left(2 k+\frac{3}{2}\right)} t^{2 k+1 / 2}, \quad t>0,
$$

from which we obtain (48) by using two of the formulas given in the following lemma.

Lemma 13. Let $k \in \mathbb{Z}_{>-1}$ and $v \in \mathbb{C}$. Then if $v \notin \mathbb{Z}_{<0 \text {, }}$

$$
\Gamma(2 k+v+1)=\Gamma(v+1)(v+1)_{2 k}=4^{k} \Gamma(v+1)\left(\frac{v+1}{2}\right)_{k}\left(\frac{1}{2} v+1\right)_{k}
$$

and if $v-\frac{1}{2} \notin \mathbb{Z}_{<0}$,

$$
\Gamma\left(2 k+v+\frac{1}{2}\right)=\Gamma\left(v+\frac{1}{2}\right)\left(v+\frac{1}{2}\right)_{2 k}=4^{k} \Gamma\left(v+\frac{1}{2}\right)\left(\frac{2 v+1}{4}\right)_{k}\left(\frac{2 v+3}{4}\right)_{k} .
$$

In particular, we have the following:

$$
\Gamma(2 k+1)=(2 k) !=4^{k} k !\left(\frac{1}{2}\right)_{k}, \quad \Gamma(2 k+2)=(2 k+1) !=4^{k} k !\left(\frac{3}{2}\right)_{k} .
$$




\subsection{Green's Function $G_{y}(t, \tau)$ for Equation (19)}

Corresponding to Equations (24) and (25), we define the Green's function $G_{y}(t, \tau)$ for Equation (19), so that it satisfies the following:

$$
\begin{aligned}
\frac{d}{d t} G_{y}(t, \tau)-a(t+b) \cdot G_{y}(t, \tau) & =\delta_{\epsilon_{1}}(t-\tau), \\
G_{y}(t, \tau)-a \cdot{ }_{R} D_{t}^{-1}\left[(t+b) \cdot G_{y}(t, \tau)\right] & =H_{\epsilon_{1}}(t-\tau) .
\end{aligned}
$$

Lemma 14. Let $y_{1}(t)$ be given by (44), and $G_{y}(t, \tau)$ satisfy (53) and (54). Then in the limit $\epsilon_{1} \rightarrow 0, G_{y}(t, \tau)$ tends to $G_{y, 0}(t, \tau)$, which is given by the following:

$$
\begin{aligned}
G_{y, 0}(t, \tau) & =\frac{y_{1}(t)}{y_{1}(\tau)} H(t-\tau)=e^{\frac{1}{2} a\left(t^{2}+2 b t-\tau^{2}-2 b \tau\right)} H(t-\tau) \\
& =\sum_{k=0}^{\infty} a^{k} \sum_{l=0}^{k} \frac{1}{2^{l} l !(k-l) !}(\tau+b)^{k-l}(t-\tau)^{k+l} H(t-\tau) .
\end{aligned}
$$

When $\tau=0$, we have $G_{y, 0}(t, 0)=y_{1}(t) H(t)$ in the limit, with (44) or (45).

Proof. Following Lemma $5, G_{y, 0}(t, \tau)$ is chosen to be the complementary solution of $G_{y, 0}(t, \tau)=1$ at $t>\tau$. The third member in Equation (55) is expressed as the following:

$$
\begin{aligned}
e^{\frac{1}{2} a\left(t^{2}+2 b t-\tau^{2}-2 b \tau\right)} & =\sum_{k=0}^{\infty} \frac{a^{k}}{2^{k} k !}(t-\tau)^{k}(t+\tau+2 b)^{k} \\
& =\sum_{k=0}^{\infty} \frac{a^{k}}{2^{k} k !}(t-\tau)^{k} \sum_{l=0}^{k} \frac{k !}{l !(k-l) !}(t-\tau)^{l}(2 \tau+2 b)^{k-l}, \quad t>\tau .
\end{aligned}
$$

Lemma 15. Let Condition 2 (i) be satisfied, and $G_{y, 0}(t, \tau)$ be given by (55). Then Theorem 1 shows that a particular solution of Equation (19) is given by $y_{f}(t)=\int_{0}^{t} G_{y, 0}(t, \tau) \tilde{f}(\tau) d \tau$.

3.3. Green's Function $G_{u}(t, \tau)$ for Equation (18)

Corresponding to Equations (24) and (25), we define the Green's function $G_{u}(t, \tau)$ for Equation (18), so that it satisfies the following:

$$
\begin{aligned}
{ }_{R} D_{t}^{3 / 2} G_{u}(t, \tau)-a(t+b) \cdot{ }_{R} D_{t}^{1 / 2} G_{u}(t, \tau) & =\delta_{\epsilon_{1}}(t-\tau), \\
{ }_{R} D_{t}^{1 / 2} G_{u}(t, \tau)-a \cdot{ }_{R} D_{t}^{-1}\left[(t+b) \cdot{ }_{R} D_{t}^{1 / 2} G_{u}(t, \tau)\right] & =H_{\epsilon_{1}}(t-\tau) .
\end{aligned}
$$

We note that these equations are obtained from Equations (53) and (54), by replacing $G_{y}(t, \tau)$ by ${ }_{R} D_{t}^{1 / 2} G_{u}(t, \tau)$. As a consequence, we obtain the following lemma by using Lemma 14 .

Lemma 16. Let $G_{y, 0}(t, \tau)$ be given by (55), and $G_{u}(t, \tau)$ satisfy (57) and (58). Then in the limit $\epsilon_{1} \rightarrow 0, G_{u}(t, \tau)$ tends to $G_{u, 0}(t, \tau)={ }_{R} D_{t}^{-1 / 2} G_{y, 0}(t, \tau)$, which is given by the following:

$$
\begin{aligned}
G_{u, 0}(t, \tau)= & \sum_{k=0}^{\infty} a^{k} \sum_{l=0}^{k} \frac{1}{2^{l} l !(k-l) !} \frac{(k+l) !}{\Gamma\left(k+l+\frac{3}{2}\right)}(\tau+b)^{k-l}(t-\tau)^{k+l+1 / 2} H(t-\tau) \\
= & {\left[\frac{(t-\tau)^{1 / 2}}{\Gamma\left(\frac{3}{2}\right)}+a \tau_{1} \frac{(t-\tau)^{3 / 2}}{\Gamma\left(\frac{5}{2}\right)}+a\left(1+a \tau_{1}^{2}\right) \frac{(t-\tau)^{5 / 2}}{\Gamma\left(\frac{7}{2}\right)}\right.} \\
& \left.+O\left((t-\tau)^{7 / 2}\right)\right] H(t-\tau),
\end{aligned}
$$


where $\tau_{1}=\tau+b$. When $\tau=0$, we have $G_{u, 0}(t, 0)=u_{1}(t) H(t)$ in the limit, with (47) or (48).

Remark 6. The Green's function given by (59) for $b=0$ is not in agreement with the first several terms obtained in [14], except the leading term.

Lemma 17. Let Condition 2 (i) be satisfied, and $G_{u, 0}(t, \tau)$ be given by (59). Then, Theorem 1 shows that a particular solution of Equation (18) is given by the following: $u_{f}(t)=\int_{0}^{t} G_{u, 0}(t, \tau) \tilde{f}(\tau) d \tau$.

\subsection{Solution of Integral Equations (54) and (58), by Iterations}

We put $t=\tau+x, \tau_{1}=\tau+b$ and $\tilde{y}(x)=G_{y}(\tau+x, \tau)$ in Equations (53) and (54), and then we have the following:

$$
\begin{aligned}
\frac{d}{d x} \tilde{y}(x)-a\left(\tau_{1}+x\right) \cdot \tilde{y}(x) & =\delta_{\epsilon_{1}}(x), \\
\tilde{y}(x)-a \cdot{ }_{R} D_{x}^{-1}\left[\left(\tau_{1}+x\right) \cdot \tilde{y}(x)\right] & =H_{\epsilon_{1}}(x) .
\end{aligned}
$$

We put $t=\tau+x, \tau_{1}=\tau+b$ and $\tilde{u}(x)=G_{u}(\tau+x, \tau)$ in Equations (57) and (58), and then we have the following:

$$
\begin{aligned}
{ }_{R} D_{x}^{3 / 2} \tilde{u}(x)-a\left(\tau_{1}+x\right) \cdot{ }_{R} D_{x}^{1 / 2} \tilde{u}(x) & =\delta_{\epsilon_{1}}(x), \\
{ }_{R} D_{x}^{1 / 2} \tilde{u}(x)-a \cdot{ }_{R} D_{x}^{-1}\left[\left(\tau_{1}+x\right) \cdot{ }_{R} D_{x}^{1 / 2} \tilde{u}(x)\right] & =H_{\epsilon_{1}}(x) .
\end{aligned}
$$

We note that these equations are obtained from Equations (60) and (61), by replacing $\tilde{y}(x)$ by ${ }_{R} D_{x}^{1 / 2} \tilde{u}(x)$.

In [14], the solution of (62) is obtained by transforming it to an integral equation and then solving it by iterations. By (63), the integral equation is the following:

$$
\tilde{u}(x)={ }_{R} D_{x}^{-1 / 2} H_{\epsilon_{1}}(x)+a \cdot{ }_{R} D_{x}^{-1 / 2} \cdot{ }_{R} D_{x}^{-1}\left[\left(\tau_{1}+x\right) \cdot{ }_{R} D_{x}^{1 / 2} \tilde{u}(x)\right] .
$$

In the case of Equation (60), the integral equation is obtained with the aid of (61), as follows:

$$
\tilde{y}(x)=H_{\epsilon_{1}}(x)+a \cdot{ }_{R} D_{x}^{-1}\left[\left(\tau_{1}+x\right) \cdot \tilde{y}(x)\right] .
$$

This integral equation is an example of the type of equation which was discussed in the book [13].

Remark 7. By the operational calculus described in [11-13], applied to (65), the particular solution of (60) is given by the following:

$$
\tilde{y}(x)=\frac{1}{1-a \cdot{ }_{R} D_{x}^{-1}\left(\tau_{1}+x\right)} H_{\epsilon_{1}}(x)=\left\{1+\sum_{k=1}^{\infty} a^{k}\left[{ }_{R} D_{x}^{-1}\left(\tau_{1}+x\right)\right]^{k}\right\} H_{\epsilon_{1}}(x) .
$$

We can write this as the following:

$$
\tilde{y}(x)=\sum_{k=0}^{\infty} a^{k} \tilde{y}_{k}(x),
$$

where

$$
\tilde{y}_{0}(x)=H_{\epsilon_{1}}(x), \quad \tilde{y}_{k+1}(x)={ }_{R} D_{x}^{-1}\left[\left(\tau_{1}+x\right) \tilde{y}_{k}(x)\right], \quad k \in \mathbb{Z}_{>-1} .
$$

We can regard the solution given by (67) with (68), as the solution of Equation (65) obtained by iterations. 
Lemma 18. By using (68), we obtain the following:

$$
\tilde{y}_{k}(x) \simeq \frac{1}{k !}\left(\tau_{1} x+\frac{1}{2} x^{2}\right)^{k} H_{\epsilon_{1}}(x) .
$$

By using (67) with (69), in the limit $\epsilon_{1} \rightarrow 0$, we obtain the following: $\tilde{y}(x)=G_{y, 0}(\tau+x, \tau)$ given in Lemma 14.

Proof. We show that $\tilde{y}_{k}(t)$ given by (69) satisfies (68) for $k \in \mathbb{Z}_{>-1}$. By using (69), with the aid of Lemma 4, we obtain the following:

$$
\begin{aligned}
& \left.{ }_{R} D_{x}^{-1}\left[\tau_{1}+x\right) \tilde{y}_{k}(x)\right] \simeq{ }_{R} D_{x}^{-1}\left[f_{1}^{\prime}(x) f_{k}(x) H_{\epsilon_{1}}(x)\right]={ }_{R} D_{x}^{-1}\left\{f_{k+1}^{\prime}(x) H_{\epsilon_{1}}(x)\right\} \\
& =f_{k+1}(x) H_{\epsilon_{1}}(x)-{ }_{R} D_{x}^{-1}\left\{f_{k+1}(x) \delta_{\epsilon_{1}}(x)\right\} \simeq f_{k+1}(x) H_{\epsilon_{1}}(x)=\tilde{y}_{k+1}(x)
\end{aligned}
$$

where $f_{k}(x)=\frac{1}{k !}\left(\tau_{1} x+\frac{1}{2} x^{2}\right)^{k}$ and $f_{1}^{\prime}(x)=\tau_{1}+x$.

Remark 8. As stated in the proof of Lemma 14, $G_{y, 0}(t, \tau)$ given by (55) is the solution of Equation (54) in the limit $\epsilon_{1} \rightarrow 0$, the corresponding $\tilde{y}(x)=G_{y, 0}(\tau+x, \tau)$ is obtained by solving (61) in the limit $\epsilon_{1} \rightarrow 0$. The solution of that equation is obtained by using Equations (67)-(70) in the limit $\epsilon_{1} \rightarrow 0$, by deleting the fourth member in (70).

In the case of Equation (62), in place of Remark 7, we have the following remark.

Remark 9. By the operational calculus applied to (64), the particular solution of (62) is given by the following:

$$
\begin{aligned}
\tilde{u}(x) & =\frac{1}{1-a \cdot{ }_{R} D_{x}^{-3 / 2}\left(\tau_{1}+x\right)_{R} D_{x}^{1 / 2}}{ }_{R} D_{x}^{-1 / 2} H_{\epsilon_{1}}(x) \\
& =\left\{1+\sum_{k=1}^{\infty} a^{k}\left[_{R} D_{x}^{-3 / 2}\left(\tau_{1}+x\right)_{R} D_{x}^{1 / 2}\right]^{k}\right\}_{R} D_{x}^{-1 / 2} H_{\epsilon_{1}}(x)
\end{aligned}
$$

We can write this as the following:

$$
\tilde{u}(x)=\sum_{k=0}^{\infty} a^{k} \tilde{u}_{k}(x),
$$

where

$$
\tilde{u}_{0}(x)={ }_{R} D_{x}^{-1 / 2} H_{\epsilon_{1}}(x), \quad \tilde{u}_{k+1}(x)={ }_{R} D_{x}^{-3 / 2}\left[\left(\tau_{1}+x\right)_{R} D_{x}^{1 / 2} \tilde{u}_{k}(x)\right], \quad k \in \mathbb{Z}_{>-1} .
$$

Since Equation (73) is obtained from Equation (69) by replacing $\tilde{y}_{k}(x)$ by ${ }_{R} D_{x}^{1 / 2} \tilde{u}_{k}(x), \tilde{u}_{k}(x)$ is related with $\tilde{y}_{k}(t)$ given by (69) by $\tilde{u}_{k}(x)={ }_{R} D_{x}^{-1 / 2} \tilde{y}_{k}(t)$.

Lemma 19. By using (72) with $\tilde{u}_{k}(x)={ }_{R} D_{x}^{-1 / 2} \tilde{y}_{k}(t)$ and (69), in the limit $\epsilon_{1} \rightarrow 0$, we obtain $\tilde{u}(x)=G_{u, 0}(\tau+x, \tau)$ given in Lemma 16 .

Remark 10. Corresponding to Remark $8, G_{u, 0}(t, \tau)$ given by (55) is the solution of Equation (58) in the limit $\epsilon_{1} \rightarrow 0$, and hence the corresponding $\tilde{u}(x)=G_{u, 0}(\tau+x, \tau)$ is obtained by solving (63) in the limit $\epsilon_{1} \rightarrow 0$. The solution of that equation is obtained by using Equation (72) with $\tilde{u}_{k}(x)={ }_{R} D_{x}^{-1 / 2} \tilde{y}_{k}(t)$ and $\tilde{y}_{k}(t)$ given in (69), in the limit $\epsilon_{1} \rightarrow 0$.

\subsection{Solution of Equations (20) and (21) Satisfying Condition 2 (iii), with the Aid of Theorem 1}

In Sections 3.1-3.4, we studied the solutions of Equations (18) and (19). In Sections 3.5, $4.2,4.3$ and 5, we study the solutions of these equations for the case of $b=0$, so that we are concerned with Equations (20) and (21). 
When Condition 2 (iii) is satisfied, Equations (20) and (21) are expressed by the following:

$$
\begin{aligned}
{ }_{R} D_{t}^{3 / 2} u(t)-a t \cdot{ }_{R} D_{t}^{1 / 2} u(t) & ={ }_{R} D_{t}^{\beta+1} H_{\epsilon}(t)=\frac{t^{-\beta-1+\epsilon}}{\Gamma(-\beta+\epsilon)} H(t), \\
\frac{d}{d t} y(t)-a t \cdot y(t) & ={ }_{R} D_{t}^{\beta+1} H_{\epsilon}(t)=\frac{t^{-\beta-1+\epsilon}}{\Gamma(-\beta+\epsilon)} H(t) .
\end{aligned}
$$

Lemma 20. When $-\beta \in \mathbb{C}_{+}$, we put $v=-\beta+\epsilon$. Then the solution of Equation (75), which is obtained with the aid of Theorem 1, is given by the following:

$$
y_{f}(t)=\frac{1}{\Gamma(v+1)} \sum_{k=0}^{\infty} \frac{a^{k}}{\left(1+\frac{v}{2}\right)_{k} 2^{k}} t^{2 k+v} H(t)=\sum_{k=0}^{\infty} \frac{(2 a)^{k}\left(\frac{1+v}{2}\right)_{k}}{\Gamma(2 k+v+1)} t^{2 k+v} H(t) .
$$

Proof. Now, the inhomogeneous term $\frac{1}{\Gamma(-\beta+\epsilon)} t^{-\beta-1+\epsilon} H(t)$ is expressed by $\frac{1}{\Gamma(v)} t^{\nu-1} H(t)$. By using $G_{y, 0}(t, \tau)$ given in Lemma 14 for $b=0$, and $\tilde{f}(t)=\frac{1}{\Gamma(v)} t^{\nu-1} H(t)$, in $y_{f}(t)$ given in Lemma 15, we have the following:

$$
\begin{aligned}
y_{f}(t) & =\int_{0}^{t} G_{y, 0}(t, \tau) \frac{\tau^{\nu-1}}{\Gamma(v)} d \tau \cdot H(t)=\int_{0}^{t} e^{\frac{1}{2} a\left(t^{2}-\tau^{2}\right)} \frac{\tau^{\nu-1}}{\Gamma(\nu)} d \tau \cdot H(t) \\
& =\sum_{k=0}^{\infty} \frac{a^{k}}{k ! 2^{k} \Gamma(v)} \int_{0}^{t}\left(t^{2}-\tau^{2}\right)^{k} \tau^{\nu-1} d \tau \cdot H(t) .
\end{aligned}
$$

By putting $\tau^{2}=t^{2} \eta$, and by using the formula $\int_{0}^{1}(1-\eta)^{\alpha_{1}-1} \eta^{\beta_{1}-1} d \eta=\frac{\Gamma\left(\alpha_{1}\right) \Gamma\left(\beta_{1}\right)}{\Gamma\left(\alpha_{1}+\beta_{1}\right)}$,

$$
\begin{aligned}
y_{f}(t) & =\sum_{k=0}^{\infty} \frac{a^{k}}{k ! 2^{k} \Gamma(v)} t^{2 k+v} \frac{1}{2} \int_{0}^{1}(1-\eta)^{k} \eta^{\frac{1}{2} v-1} d \eta \cdot H(t) \\
& =\sum_{k=0}^{\infty} \frac{a^{k}}{k ! 2^{k} \Gamma(v)} t^{2 k+v} \frac{k ! \Gamma\left(\frac{1}{2} v\right)}{2 \Gamma\left(\frac{1}{2} v+k+1\right)} H(t) .
\end{aligned}
$$

By using $\Gamma\left(\frac{1}{2} v+k+1\right)=\Gamma\left(\frac{1}{2} v+1\right)\left(\frac{1}{2} v+1\right)_{k}=\frac{1}{2} v \Gamma\left(\frac{1}{2} v\right)\left(\frac{1}{2} v+1\right)_{k}$ and Formula (50) in this equation, we have (76).

Remark 11. Here we give another expression of $y_{f}(t)$, by using (55) for $b=0$, in the second member of (77):

$$
\begin{aligned}
y_{f}(t) & =\sum_{k=0}^{\infty} \frac{a^{k}}{k ! 2^{k}} \sum_{l=0}^{k} \frac{k ! 2^{k-l}}{(k-l) ! l ! \Gamma(v)} \int_{0}^{t}(t-\tau)^{k+l} \tau^{k-l+v-1} d \tau \cdot H(t) \\
& =\sum_{k=0}^{\infty} \frac{a^{k}}{2^{k}} \sum_{l=0}^{k} \frac{2^{k-l}}{(k-l) ! l ! \Gamma(v)} \frac{(k+l) ! \Gamma(k-l+v)}{\Gamma(2 k+v+1)} t^{2 k+v} H(t) .
\end{aligned}
$$

Since this $y_{f}(t)$ must be equal to $y_{f}(t)$ given by (76), when $v=-\beta+\epsilon$, we have the following:

$$
\sum_{l=0}^{k} \frac{(k+l) ! \Gamma(k-l+v) 2^{k-l}}{(k-l) ! l ! \Gamma(v)}=4^{k}\left(\frac{1+v}{2}\right)_{k} .
$$


Lemma 21. When $-\beta \in \mathbb{C}_{+}$, the solution of Equation (74), which is obtained with the aid of Theorem 1, is given by the following:

$$
\begin{aligned}
u_{f}(t)= & \sum_{k=0}^{\infty} \frac{(2 a)^{k}\left(\frac{1-\beta+\epsilon}{2}\right)_{k}}{\Gamma\left(2 k-\beta+\epsilon+\frac{3}{2}\right)} t^{2 k-\beta+1 / 2+\epsilon} H(t)=\frac{1}{\Gamma\left(\frac{3}{2}-\beta+\epsilon\right)} t^{-\beta+1 / 2+\epsilon} \\
& \times{ }_{2} F_{2}\left(1, \frac{1-\beta+\epsilon}{2} ; \frac{3-2 \beta+2 \epsilon}{4}, \frac{5-2 \beta+2 \epsilon}{4} ; \frac{1}{2} a t^{2}\right) H(t) .
\end{aligned}
$$

Proof. By using $G_{u, 0}(t, \tau)$ given in Lemma 16 for $b=0$, and $\tilde{f}(t)=\frac{1}{\Gamma(v)} t^{\nu-1} H(t)$, in $u_{f}(t)$ given in Lemma 17, we have the following:

$$
\begin{aligned}
u_{f}(t) & =\int_{0}^{t} G_{u, 0}(t, \tau) \frac{\tau^{v-1}}{\Gamma(v)} d \tau \cdot H(t) \\
& =\sum_{k=0}^{\infty} \frac{a^{k}}{2^{k}} \sum_{l=0}^{k} \frac{(k+l) ! 2^{k-l}}{(k-l) ! l ! \Gamma\left(k+l+\frac{3}{2}\right) \Gamma(v)} \int_{0}^{t}(t-\tau)^{k+l+1 / 2} \tau^{k-l+v-1} d \tau \cdot H(t) \\
& =\sum_{k=0}^{\infty} \frac{a^{k}}{2^{k}} \sum_{l=0}^{k} \frac{(k+l) ! 2^{k-l}}{(k-l) ! l ! \Gamma(v)} \frac{\Gamma(k-l+v)}{\Gamma\left(2 k+v+\frac{3}{2}\right)} t^{2 k+v+1 / 2} H(t) .
\end{aligned}
$$

By using (80) in this equation, when $v=-\beta+\epsilon$, we have (81). The last equality in (81) is due to Formula (51) for $v=1-\beta+\epsilon$.

\section{Solution of Equations (22), (75) and (74) Satisfying Condition 2 (iii) by Iterations} 4.1. Solution of Equation (22) Satisfying Condition 2 (iii) by Iterations

Lemma 22. Let $u(t)$ be a solution of (22) and $y(t)={ }_{R} D_{t}^{\lambda-\alpha+\rho} u(t)$. Then, $y(t)$ satisfies the following:

$$
\begin{gathered}
{ }_{R} D_{t}^{-\lambda+\alpha} y(t)-a t^{\lambda} \cdot y(t)={ }_{R} D_{t}^{\beta+1} H_{\epsilon}(t), \\
y(t)-a \cdot{ }_{R} D_{t}^{\lambda-\alpha}\left[t^{\lambda} \cdot y(t)\right]={ }_{R} D_{t}^{\lambda-\alpha+\beta+1} H_{\epsilon}(t) .
\end{gathered}
$$

In order to obtain a solution, we use the method adopted in Remarks 7 and 9.

Remark 12. Let $\gamma=\alpha-\beta+\epsilon-\lambda$. Then, by the operational calculus applied to (84), the particular solution of Equation (83) is given by the following:

$$
y(t)=\frac{1}{1-a \cdot{ }_{R} D_{t}^{\lambda-\alpha} t^{\lambda}}{ }_{R} D_{t}^{-\gamma+1} H(t)=\left\{1+\sum_{k=1}^{\infty} a^{k}\left[{ }_{R} D_{t}^{\lambda-\alpha} t^{\lambda}\right]^{k}\right\}_{R} D_{t}^{-\gamma+1} H(t) .
$$

We can write this as the following:

$$
y(t)=\sum_{k=0}^{\infty} a^{k} y_{k}(t)
$$

where

$$
y_{0}(t)={ }_{R} D_{t}^{-\gamma+1} H(t), \quad y_{k+1}(t)={ }_{R} D_{t}^{\lambda-\alpha}\left[t^{\lambda} \cdot y_{k}(t)\right], \quad k \in \mathbb{Z}_{>-1} .
$$

Lemma 23. The solution of (83) is given by Equation (86) with the following:

$$
y_{k}(t)=\frac{C_{\lambda, k}(\gamma)}{\Gamma(k \alpha+\gamma)} t^{k \alpha+\gamma-1} H(t),
$$


where $C_{\lambda, k}(\gamma)=\alpha^{k}\left(\frac{\gamma}{\alpha}\right)_{k}$ if $\lambda=1$, and if $\lambda \in \mathbb{R}_{>0}, C_{\lambda, k}(\gamma)=1$ when $k=0$, and

$$
C_{\lambda, k}(\gamma)=\prod_{l=1}^{k-1} \frac{\Gamma(l \alpha+\gamma+\lambda)}{\Gamma(l \alpha+\gamma)}, \quad k \in \mathbb{Z}_{>0} .
$$

Proof. We show that $y_{k}(t)$ given by (88) satisfies (87). By using (88), we have the following:

$$
\begin{aligned}
{ }_{R} D_{t}^{\lambda-\alpha}\left[t^{\lambda} \cdot y_{k}(t)\right] & ={ }_{R} D_{t}^{\lambda-\alpha}\left[t^{\lambda} \cdot \frac{C_{\lambda, k}(\gamma)}{\Gamma(k \alpha+\gamma)} t^{k \alpha+\gamma-1} H(t)\right] \\
& =\frac{C_{\lambda, k}(\gamma)}{\Gamma(k \alpha+\gamma)} \cdot \frac{\Gamma(k \alpha+\gamma+\lambda)}{\Gamma((k+1) \alpha+\gamma)} t^{(k+1) \alpha+\gamma-1} H(t) .
\end{aligned}
$$

By using (89), we confirm that (90) gives (88) with $k$ replaced by $k+1$. In order to show that when $\lambda=1$, (89) becomes $C_{\lambda, k}(\gamma)=\alpha^{k}\left(\frac{\gamma}{\alpha}\right)_{k}$, we use the following formula:

$$
\frac{\Gamma(k \alpha+\gamma+1)}{\Gamma(k \alpha+\gamma)}=k \alpha+\gamma=\alpha\left(\frac{\gamma}{\alpha}+k\right) .
$$

By using Lemmas 22 and 23, we obtain the following lemma.

Lemma 24. Let $C_{\lambda, k}(\gamma)$ be given as in Lemma 23. Then the solution of (22) is given by the following:

$$
u(t)={ }_{R} D_{t}^{\alpha-\rho-1} y(t)=\sum_{k=0}^{\infty} \frac{a^{k} C_{\lambda, k}(\alpha-\beta+\epsilon-\lambda)}{\Gamma(k \alpha-\beta+\rho-\lambda+\epsilon+1)} t^{k \alpha-\beta+\rho-\lambda+\epsilon} H(t) .
$$

\subsection{Solution of Equation (75) by Iterations}

Equation (75) is Equation (22) for $\rho=1, \lambda=1$ and $\alpha=2$. By using Lemma 24, we obtain the following lemma.

Lemma 25. The solution of Equation (75) is given by the following:

$$
\begin{aligned}
y(t) & =\sum_{k=0}^{\infty} \frac{(2 a)^{k}\left(\frac{1-\beta+\epsilon}{2}\right)_{k}}{\Gamma(2 k-\beta+\epsilon+1)} t^{2 k-\beta+\epsilon} H(t) \\
& =\frac{1}{\Gamma(1-\beta+\epsilon)} \sum_{k=0}^{\infty} \frac{a^{k}}{\left(1+\frac{-\beta+\epsilon}{2}\right)_{k} 2^{k}} t^{2 k-\beta+\epsilon} H(t) .
\end{aligned}
$$

This agrees with (76), which is derived for the case of $-\beta \in \mathbb{C}_{+}$.

Proof. The second equality of (93) is due to Formula (50) for $v=-\beta+\epsilon$.

Remark 13. When $\beta=0$ and $\epsilon=\epsilon_{1}, y(t)$, satisfying Equation (75), is $G_{y}(t, 0)$, satisfying (53) for $\tau=0$ and $b=0$. In this case, Equation (93) gives the following:

$$
y(t)=G_{y}(t, 0)=\sum_{k=0}^{\infty} \frac{(2 a)^{k}\left(\frac{1}{2}\right)_{k}}{(2 k) !} t^{2 k+\epsilon_{1}} H(t)=\sum_{k=0}^{\infty} \frac{a^{k}}{k ! 2^{k}} t^{2 k+\epsilon_{1}} H(t)=e^{\frac{1}{2} a t^{2}} t^{\epsilon_{1}} H(t),
$$

which tends to $G_{y, 0}(t, 0)$ in the limit $\epsilon_{1} \rightarrow 0$, as stated in Lemma 14.

\subsection{Solution of Equation (74) by Iterations}

Equation (74) is Equation (22) for $\rho=\frac{3}{2}, \lambda=1$ and $\alpha=2$. By using Lemma 24, we obtain the following lemma. 
Lemma 26. The solution of Equation (74) is given by the following:

$$
\begin{aligned}
u(t)= & \sum_{k=0}^{\infty} \frac{(2 a)^{k}\left(\frac{1-\beta+\epsilon}{2}\right)_{k}}{\Gamma\left(2 k-\beta+\epsilon+\frac{3}{2}\right)} t^{2 k-\beta+1 / 2+\epsilon} H(t)=\frac{1}{\Gamma\left(\frac{3}{2}-\beta+\epsilon\right)} t^{-\beta+1 / 2+\epsilon} \\
& \times{ }_{2} F_{2}\left(1, \frac{1-\beta+\epsilon}{2} ; \frac{3-2 \beta+2 \epsilon}{4}, \frac{5-2 \beta+2 \epsilon}{4} ; \frac{1}{2} a t^{2}\right) H(t) .
\end{aligned}
$$

This agrees with (81), which is derived for the case of $-\beta \in \mathbb{C}_{+}$.

Proof. The last equality in (81) is due to Formula (51) for $v=1-\beta+\epsilon$.

Remark 14. When $\beta=0$ and $\epsilon=\epsilon_{1}, u(t)$ satisfying Equation (74) is $G_{u}(t, 0)$, satisfying (57) for $\tau=0$ and $b=0$. In this case, Equation (95) gives the following:

$$
u(t)=G_{u}(t, 0)=\frac{1}{\Gamma\left(\frac{3}{2}\right)} t^{1 / 2+\epsilon_{1}} \cdot{ }_{2} F_{2}\left(1, \frac{1}{2} ; \frac{3}{4}, \frac{5}{4} ; \frac{1}{2} a t^{2}\right) H(t),
$$

which tends to $G_{u, 0}(t, 0)$ in the limit $\epsilon_{1} \rightarrow 0$, as stated in Lemma 16.

\section{Solution of Equations (20) and (21) by Theorems 2 and 3}

\subsection{Transformed Differential Equations of Equations (20) and (21)}

We construct the transformed differential equations of Equations (20) and (21), which appear in Theorems 2 and 3. For this purpose, we use the following formula.

Lemma 27. Let $\lambda \in \mathbb{C}_{+}, m \in \mathbb{Z}_{>-1}$ and $\rho=m-\lambda$. Then, we have the following:

$$
{ }_{R} D_{t}^{\rho}[t u(t)]=t \cdot{ }_{R} D_{t}^{\rho} u(t)+\rho \cdot{ }_{R} D_{t}^{\rho-1} u(t) .
$$

Proof. When $m=0$ and $\rho=-\lambda$, this is confirmed with the aid of Formula (2) as follows:

$$
\begin{aligned}
& { }_{R} D_{t}^{-\lambda}[t u(t)]=\frac{1}{\Gamma(\lambda)} \int_{-\infty}^{t}(t-\xi)^{\lambda-1} \xi u(\xi) d \xi \\
& =\frac{1}{\Gamma(\lambda)} \int_{-\infty}^{t}(t-\xi)^{\lambda-1}(t-(t-\xi)) u(\xi) d \xi=t \cdot{ }_{R} D_{t}^{-\lambda} u(t)-\lambda \cdot{ }_{R} D_{t}^{-\lambda-1} u(t) .
\end{aligned}
$$

We prove (97) by mathematical induction. In fact, when (97) holds for a value of $m$, we confirm it to hold, even when $\rho=m+\lambda$ is replaced by $\rho+1$, by applying $\frac{d}{d t}$ to (97).

Remark 15. When $u(t)=\frac{t^{v+\epsilon}}{\Gamma(v+\epsilon+1)} H(t)$, by using (9), we confirm (97) as follows:

$$
\begin{aligned}
& { }_{R} D_{t}^{\rho}[t u(t)]={ }_{R} D_{t}^{\rho}\left[t \frac{t^{v+\epsilon}}{\Gamma(v+\epsilon+1)} H(t)\right]=(v+\epsilon+1)_{R} D_{t}^{\rho}\left[\frac{t^{v+\epsilon+1}}{\Gamma(v+\epsilon+2)} H(t)\right] \\
& =((v+\epsilon+1-\rho)+\rho) \frac{t^{v+\epsilon-\rho+1}}{\Gamma(v+\epsilon-\rho+2)} H(t)=t \cdot{ }_{R} D_{t}^{\rho} u(t)+\rho \cdot{ }_{R} D_{t}^{\rho-1} u(t) .
\end{aligned}
$$

With the aid of Lemma 27, we obtain the transformation of Equation (20) as follows:

$$
\begin{aligned}
\tilde{p}_{F}\left(t,{ }_{R} D_{t}\right) w(t) & :={ }_{R} D_{t}^{-\beta} p_{F}\left(t,{ }_{R} D_{t}\right)_{R} D_{t}^{\beta} w(t)={ }_{R} D_{t}^{-\beta}\left[{ }_{R} D_{t}^{3 / 2}-a t \cdot{ }_{R} D_{t}^{1 / 2}\right]_{R} D_{t}^{\beta} w(t) \\
& ={ }_{R} D_{t}^{3 / 2} w(t)-a t \cdot{ }_{R} D_{t}^{1 / 2} w(t)+a \beta \cdot{ }_{R} D_{t}^{-1 / 2} w(t)=\tilde{f}_{\beta}(t) .
\end{aligned}
$$


When we put $\frac{d}{d t} v(t)={ }_{R} D_{t}^{1 / 2} w(t)$ and hence $v(t)={ }_{R} D_{t}^{-1 / 2} w(t)$ in (99), we have the following equation for $v(t)$, which is a transformation of (21):

$$
\begin{aligned}
\tilde{p}_{L}\left(t,{ }_{R} D_{t}\right) \frac{d}{d t} v(t) & :={ }_{R} D_{t}^{-\beta} p_{L}\left(t,{ }_{R} D_{t}\right)_{R} D_{t}^{\beta} \frac{d}{d t} v(t)={ }_{R} D_{t}^{-\beta}\left[\frac{d}{d t}-a t\right]_{R} D_{t}^{\beta} \frac{d}{d t} v(t) \\
& =\frac{d^{2}}{d t^{2}} v(t)-a t \frac{d}{d t} v(t)+a \beta v(t)=\tilde{f}_{\beta}(t) .
\end{aligned}
$$

Remark 16. This equation for $a=1, \beta=n \in \mathbb{Z}_{>-1}$ and $\tilde{f}_{\beta}(t)=0$, is the differential equation satisfied by the Hermite function [15] (Chaper V, Section 2).

We put $x=t^{2}$ and $y(x)=v(t)$ in (100), and then we have

$$
x \frac{d^{2}}{d x^{2}} y(x)+\left(\frac{1}{2}-\frac{a}{2} x\right) \frac{d}{d x} y(x)+\frac{a \beta}{4} y(x)=\frac{1}{4} \tilde{f}_{\beta}(\sqrt{x}) .
$$

5.2. Complementary Solutions of Equations (99) and (100)

Lemma 28. The complementary solutions of Equation (101) are given by the following:

$$
\begin{aligned}
& y_{1}(x)={ }_{1} F_{1}\left(-\frac{\beta}{2} ; \frac{1}{2} ; \frac{1}{2} a x\right), \quad x>0, \\
& y_{2}(x)=x^{1 / 2} \cdot{ }_{1} F_{1}\left(\frac{1-\beta}{2} ; \frac{3}{2} ; \frac{1}{2} a x\right), \quad x>0,
\end{aligned}
$$

where ${ }_{1} F_{1}\left(\alpha_{1} ; \beta_{1} ; z\right)=\sum_{k=0}^{\infty} \frac{\left(\alpha_{1}\right)_{k}}{k !\left(\beta_{1}\right)_{k}} z^{k}[15]$ (Chaper VI, Section 1).

Lemma 29. The complementary solutions of Equation (100) are given by the following:

$$
\begin{aligned}
v_{1}(t)=y_{1}\left(t^{2}\right) & ={ }_{1} F_{1}\left(-\frac{\beta}{2} ; \frac{1}{2} ; \frac{1}{2} a t^{2}\right)=\sum_{k=0}^{\infty} \frac{\left(-\frac{\beta}{2}\right)_{k} a^{k}}{k !\left(\frac{1}{2}\right)_{k} 2^{k}} t^{2 k} \\
& =\sum_{k=0}^{\infty} \frac{\left(-\frac{\beta}{2}\right)_{k}(2 a)^{k}}{\Gamma(2 k+1)} t^{2 k}, \quad t>0, \\
v_{2}(t)=y_{2}\left(t^{2}\right) & =t \cdot{ }_{1} F_{1}\left(\frac{1-\beta}{2} ; \frac{3}{2} ; \frac{1}{2} a t^{2}\right)=\sum_{k=0}^{\infty} \frac{\left(\frac{1-\beta}{2}\right)_{k} a^{k}}{k !\left(\frac{3}{2}\right)_{k} 2^{k}} t^{2 k+1} \\
& =\sum_{k=0}^{\infty} \frac{\left(\frac{1-\beta}{2}\right)_{k}(2 a)^{k}}{\Gamma(2 k+2)} t^{2 k+1}, \quad t>0 .
\end{aligned}
$$

Lemma 30. The complementary solutions of Equation (99) are given by the following:

$$
\begin{aligned}
w_{1}(t) & ={ }_{R} D_{t}^{1 / 2} v_{1}(t)=\sum_{k=0}^{\infty} \frac{\left(-\frac{\beta}{2}\right)_{k}(2 a)^{k}}{\Gamma\left(2 k+\frac{1}{2}\right)} t^{2 k-1 / 2}=\sum_{k=0}^{\infty} \frac{\left(-\frac{\beta}{2}\right)_{k}(2 a)^{k}}{\Gamma\left(\frac{1}{2}\right)\left(\frac{1}{4}\right)_{k}\left(\frac{3}{4}\right)_{k}} t^{2 k-1 / 2} \\
& =\frac{1}{\Gamma\left(\frac{1}{2}\right)} t^{-1 / 2} \cdot{ }_{2} F_{2}\left(1,-\frac{\beta}{2} ; \frac{1}{4}, \frac{3}{4} ; \frac{a}{2} t^{2}\right), \quad t>0, \\
w_{2}(t) & ={ }_{R} D_{t}^{1 / 2} v_{2}(t)=\sum_{k=0}^{\infty} \frac{\left(\frac{1-\beta}{2}\right)_{k}(2 a)^{k}}{\Gamma\left(2 k+\frac{3}{2}\right)} t^{2 k+1 / 2}=\sum_{k=0}^{\infty} \frac{\left(\frac{1-\beta}{2}\right)_{k}(2 a)^{k}}{\Gamma\left(\frac{3}{2}\right)\left(\frac{3}{4}\right)_{k}\left(\frac{5}{4}\right)_{k}} t^{2 k+1 / 2} \\
& =\frac{1}{\Gamma\left(\frac{3}{2}\right)} t^{1 / 2} \cdot{ }_{2} F_{2}\left(1, \frac{1-\beta}{2} ; \frac{3}{4}, \frac{5}{4} ; \frac{a}{2} t^{2}\right), \quad t>0 .
\end{aligned}
$$

Proof. By using (104) and (105), with the aid of formulas given in Lemma 13, we obtain (106) and (107), respectively. 


\subsection{Green's Function $G_{v, \epsilon_{1}}(t, \tau)$ for Equation (100)}

Corresponding to Equations (36) and (37), the differential equations satisfied by the Green's function $G_{v, \epsilon_{1}}(t, \tau)$ for Equation (100) with $\beta$ replaced by $\beta-\epsilon+\epsilon_{1}$, are given by the following:

$$
\begin{aligned}
& \frac{d^{2}}{d t^{2}} G_{v, \epsilon_{1}}(t, \tau)-a t \cdot \frac{d}{d t} G_{v, \epsilon_{1}}(t, \tau)+a\left(\beta-\epsilon+\epsilon_{1}\right) \cdot G_{v, \epsilon_{1}}(t, \tau)=\delta_{\epsilon_{1}}(t-\tau), \\
& \begin{aligned}
\frac{d}{d t} G_{v, \epsilon_{1}}(t, \tau)-a \cdot{ }_{R} D_{t}^{-1}\left[t \cdot \frac{d}{d t} G_{v, \epsilon_{1}}(t, \tau)\right]+a\left(\beta-\epsilon+\epsilon_{1}\right) \cdot{ }_{R} D_{t}^{-1} G_{v, \epsilon_{1}}(t, \tau) \\
=H_{\epsilon_{1}}(t-\tau) .
\end{aligned}
\end{aligned}
$$

Lemma 31. Let $v_{1,0}(t)$ and $v_{2,0}(t)$, respectively, be given by (104) and (105) with $\beta$ replaced by $\beta-\epsilon$, so that the following holds:

$$
\begin{aligned}
& v_{1,0}(t)=\sum_{k=0}^{\infty} \frac{\left(-\frac{\beta-\epsilon}{2}\right)_{k}(2 a)^{k}}{\Gamma(2 k+1)} t^{2 k}={ }_{1} F_{1}\left(-\frac{\beta-\epsilon}{2} ; \frac{1}{2} ; \frac{1}{2} a t^{2}\right), \quad t>0, \\
& v_{2,0}(t)=\sum_{k=0}^{\infty} \frac{\left(\frac{1-\beta+\epsilon}{2}\right)_{k}(2 a)^{k}}{\Gamma(2 k+2)} t^{2 k+1}=t \cdot{ }_{1} F_{1}\left(\frac{1-\beta+\epsilon}{2} ; \frac{3}{2} ; \frac{1}{2} a t^{2}\right), \quad t>0 .
\end{aligned}
$$

and let $\tilde{v}_{\tau}(t)$ be given by the following:

$$
\tilde{v}_{\tau}(t)=\frac{1}{\psi_{\tau}^{\prime}(\tau)} \psi_{\tau}(t)=\frac{1}{\psi_{\tau}^{\prime}(\tau)} \sum_{k=1}^{\infty} \frac{1}{k !} \psi_{\tau}^{(k)}(\tau)(t-\tau)^{k},
$$

where

$$
\psi_{\tau}(t)=v_{1,0}(\tau) v_{2,0}(t)-v_{2,0}(\tau) v_{1,0}(t) .
$$

Then, $\tilde{v}_{\tau}(t)$ is a complementary solution of (108) and satisfies (109) for $t>\tau$ and $\epsilon_{1}=0$, so that $G_{v, 0}(t, \tau)=\tilde{v}_{\tau}(t) H(t-\tau)$ satisfies (108) and (109) in the limit $\epsilon_{1} \rightarrow 0$. In particular, when $\tau=0, G_{v, 0}(t, 0)=v_{2,0}(t) H(t)$.

Proof. $\tilde{v}_{\tau}(t)$ is so chosen such that $\tilde{v}_{\tau}(\tau)=0$ and $\tilde{v}_{\tau}^{\prime}(\tau)=1$. The statement for $\tau=0$ in this lemma is due to the fact that when $\tau=0, v_{2,0}(\tau)=0, v_{2,0}^{\prime}(\tau)=1, v_{1,0}(\tau)=1$, and $\tilde{v}_{\tau}(t)=\psi_{\tau}(t)=v_{2,0}(t)$.

Lemma 32. Let $b=0, G_{v, \epsilon_{1}}(t, \tau)$ satisfy (108) and (109), and $G_{v, 0}(t, \tau)$ be given in Lemma 31 . Then, if $\tilde{f}(t)$ satisfies Condition 2 (ii), Theorem 2 shows that a particular solution of Equation (19) is given by the following:

$$
y_{f}(t)={ }_{R} D_{t}^{\beta-\epsilon+1} \int_{0}^{t} G_{v, 0}(t, \tau) f_{\beta}(\tau) d \tau
$$

and if $\tilde{f}(t)$ satisfies Condition 2 (iii), Theorem 3 shows that a particular solution of Equation (19) is given by $y_{f}(t)={ }_{R} D_{t}^{\beta-\epsilon+1} G_{v, 0}(t, 0)$, and Remark 5 shows that ${ }_{R} D_{t}^{\epsilon_{1}} G_{v, \epsilon_{1}}(t, \tau)=G_{v, 0}(t, \tau)$. 


\subsection{Green's Function $G_{w, \epsilon_{1}}(t, \tau)$ for Equation (99)} ing:

We put $G_{w, \epsilon_{1}}(t, \tau)={ }_{R} D_{t}^{1 / 2} G_{v, \epsilon_{1}}(t, \tau)$ in (108) and (109), and then we have the follow-

$$
\begin{aligned}
{ }_{R} D_{t}^{3 / 2} G_{w, \epsilon_{1}}(t, \tau) & -a t \cdot{ }_{R} D_{t}^{1 / 2} G_{w, \epsilon_{1}}(t, \tau) \\
& +a\left(\beta-\epsilon+\epsilon_{1}\right) \cdot{ }_{R} D_{t}^{-1 / 2} G_{w, \epsilon_{1}}(t, \tau)=\delta_{\epsilon_{1}}(t-\tau), \\
{ }_{R} D_{t}^{1 / 2} G_{w, \epsilon_{1}}(t, \tau) & -a \cdot{ }_{R} D_{t}^{-1}\left[t \cdot{ }_{R} D_{t}^{1 / 2} G_{w, \epsilon_{1}}(t, \tau)\right] \\
& +a\left(\beta-\epsilon+\epsilon_{1}\right) \cdot{ }_{R} D_{t}^{-3 / 2} G_{w, \epsilon_{1}}(t, \tau)=H_{\epsilon_{1}}(t-\tau) .
\end{aligned}
$$

These equations show that $G_{w, \epsilon_{1}}(t, \tau)$ is the Green's function for Equation (99) with $\beta$ replaced by $\beta-\epsilon+\epsilon_{1}$.

By this construction, if $G_{v, \epsilon_{1}}(t, \tau)$ satisfies (108) and (109), $G_{w, \epsilon_{1}}(t, \tau)={ }_{R} D_{t}^{1 / 2} G_{v, \epsilon_{1}}(t, \tau)$ satisfies (115) and (116). As a consequence, by using Lemma 31, we have the following lemma.

Lemma 33. Let $w_{1,0}(t)$ and $w_{2,0}(t)$, respectively, be given by (106) and (107) with $\beta$ replaced by $\beta-\epsilon$ so that the following holds:

$$
\begin{aligned}
w_{1,0}(t) & ={ }_{R} D_{t}^{1 / 2} v_{1,0}(t)=\sum_{k=0}^{\infty} \frac{\left(-\frac{\beta-\epsilon}{2}\right)_{k}(2 a)^{k}}{\Gamma\left(2 k+\frac{1}{2}\right)} t^{2 k-1 / 2} \\
& =\frac{1}{\Gamma\left(\frac{1}{2}\right)} t^{-1 / 2} \cdot{ }_{2} F_{2}\left(1,-\frac{\beta-\epsilon}{2} ; \frac{1}{4}, \frac{3}{4} ; \frac{a}{2} t^{2}\right), \quad t>0 \\
w_{2,0}(t) & ={ }_{R} D_{t}^{1 / 2} v_{2,0}(t)=\sum_{k=0}^{\infty} \frac{\left(\frac{1-\beta+\epsilon}{2}\right)_{k}(2 a)^{k}}{\Gamma\left(2 k+\frac{3}{2}\right)} t^{2 k+1 / 2} \\
& =\frac{1}{\Gamma\left(\frac{3}{2}\right)} t^{1 / 2} \cdot{ }_{2} F_{2}\left(1, \frac{1-\beta+\epsilon}{2} ; \frac{3}{4}, \frac{5}{4} ; \frac{a}{2} t^{2}\right), \quad t>0,
\end{aligned}
$$

and $\tilde{v}_{\tau}(t)$ and $\psi_{\tau}(t)$ be given by (112) and (113), respectively. Then $\tilde{w}_{\tau}(t)$ is given by the following:

$$
\tilde{w}_{\tau}(t)={ }_{R} D_{t}^{1 / 2} \tilde{v}_{\tau}(t)=\frac{1}{\psi_{\tau}^{\prime}(\tau)}\left[v_{1,0}(\tau) w_{2,0}(t)-v_{2,0}(\tau) w_{1,0}(t)\right],
$$

and is a complementary solution of (115) and satisfies (116) for $t>\tau$ in the limit $\epsilon_{1} \rightarrow 0$, so that $G_{w, 0}(t, \tau)=\tilde{w}_{\tau}(t) H(t-\tau)$ satisfies (115) and (116) in the limit $\epsilon_{1} \rightarrow 0$. In particular, when $\tau=0, G_{w, 0}(t, 0)={ }_{R} D_{t}^{1 / 2} v_{2,0}(t) H(t)=w_{2,0}(t) H(t)$.

Lemma 34. Let $b=0, G_{w, \epsilon_{1}}(t, \tau)$ satisfy (115) and (116), and $G_{w, 0}(t, \tau)$ be given in Lemma 33. Then if $\tilde{f}(t)$ satisfy Condition 2 (ii), Theorem 2 shows that a particular solution of Equation (18) is given by the following:

$$
u_{f}(t)={ }_{R} D_{t}^{\beta-\epsilon} \int_{0}^{t} G_{w, 0}(t, \tau) f_{\beta}(\tau) d \tau
$$

and if $\tilde{f}(t)$ satisfies Condition 2 (iii), Theorem 3 shows that a particular solution of Equation (18) is given by $u_{f}(t)={ }_{R} D_{t}^{\beta-\epsilon} G_{w, 0}(t, 0)$, and Remark 5 shows that ${ }_{R} D_{t}^{\epsilon_{1}} G_{w, \epsilon_{1}}(t, \tau)=G_{w, 0}(t, \tau)$.

5.5. Green's Functions $G_{v, \epsilon_{1}}(t, 0)$ and $G_{w, \epsilon_{1}}(t, 0)$ for Equations (100) and (99) Obtained by Frobenius' Method

In Sections 5.3 and 5.4, the Green's functions $G_{v, \epsilon_{1}}(t, 0)$ and $G_{w, \epsilon_{1}}(t, 0)$ are given. In this section, we derive them by solving Equations (108) and (115) for $\tau=0$, by Frobenius' method. 
In this method, we assume that $G_{v, \epsilon_{1}}(t, 0)$ is expressed by the following:

$$
G_{v, \epsilon_{1}}(t, 0)=\sum_{k=0}^{\infty} p_{k} \frac{1}{\Gamma(\alpha+2 k+1)} t^{\alpha+2 k} H(t),
$$

where $p_{k}$ are constants, and $p_{0} \neq 0$. By using this and $\tau=0$ in Equation (108), we obtain the following:

$$
\begin{aligned}
& \sum_{k=0}^{\infty} p_{k}\left[\frac{t^{\alpha+2 k-2}}{\Gamma(\alpha+2 k-1)}-a\left(\alpha+2 k-\beta+\epsilon-\epsilon_{1}\right) \frac{t^{\alpha+2 k}}{\Gamma(\alpha+2 k+1)}\right] H(t) \\
& =p_{0} \frac{t^{\alpha-2}}{\Gamma(\alpha-1)} H(t)+\sum_{k=1}^{\infty}\left[p_{k}-a p_{k-1}\left(\alpha+2 k-2-\beta+\epsilon-\epsilon_{1}\right)\right] \frac{t^{\alpha+2 k-2}}{\Gamma(\alpha+2 k-1)} H(t) \\
& =\frac{t^{\epsilon_{1}-1}}{\Gamma\left(\epsilon_{1}\right)} H(t) .
\end{aligned}
$$

From this, we have $\alpha=1+\epsilon_{1}$ and the following:

$$
p_{0}=1, \quad p_{k}=2 a p_{k-1}\left(k-1+\frac{1+\epsilon-\beta}{2}\right)=(2 a)^{k}\left(\frac{1+\epsilon-\beta}{2}\right)_{k}, k \in \mathbb{Z}_{>0} .
$$

By using (123), (50) and $\alpha=1+\epsilon_{1}$ in (121), we obtain the following:

$$
\begin{aligned}
G_{v, \epsilon_{1}}(t, 0) & =\sum_{k=0}^{\infty} \frac{(2 a)^{k}\left(\frac{1-\beta+\epsilon}{2}\right)_{k}}{\Gamma\left(2 k+\epsilon_{1}+2\right)} t^{2 k+1+\epsilon_{1}} H(t) \\
& =\frac{1}{\Gamma\left(2+\epsilon_{1}\right)} \sum_{k=0}^{\infty} \frac{a^{k}\left(\frac{1-\beta+\epsilon}{2}\right)_{k}}{2^{k}\left(1+\frac{\epsilon_{1}}{2}\right)_{k}\left(\frac{3+\epsilon_{1}}{2}\right)_{k}} t^{2 k+\epsilon_{1}+1} H(t)
\end{aligned}
$$

By using the relation $G_{v, 0}(t, \tau)={ }_{R} D_{t}^{\epsilon_{1}} G_{v, \epsilon_{1}}(t, \tau)$ given in Lemma 32 to (124), or by replacing $\epsilon_{1}$ by 0 in (124), we obtain the expression for $G_{v, 0}(t, 0)=v_{2,0}(t) H(t)$ given in Lemma 31.

In the case of Equation (115), we note that it is obtained from Equation (108) by replacing ${ }_{R} D_{t}^{1 / 2} G_{v, \epsilon_{1}}(t, \tau)$ by $G_{w, \epsilon_{1}}(t, \tau)$. In place of $(121)$, we use the following:

$$
G_{w, \epsilon_{1}}(t, 0)=\sum_{k=0}^{\infty} p_{k} \frac{1}{\Gamma\left(\alpha+2 k+\frac{1}{2}\right)} t^{\alpha+2 k-1 / 2} H(t),
$$

in Equation (115) for $\tau=0$; we then obtain $\alpha=1-\epsilon_{1}$, and (123). By using these and (51) in (125), we have the following:

$$
\begin{aligned}
G_{w, \epsilon_{1}}(t, 0) & =\sum_{k=0}^{\infty} \frac{2^{k} a^{k}\left(\frac{1-\beta+\epsilon}{2}\right)_{k}}{\Gamma\left(2 k+\epsilon_{1}+\frac{3}{2}\right)} t^{2 k+\epsilon_{1}+1 / 2} H(t) \\
& =\frac{1}{\Gamma\left(\frac{3}{2}+\epsilon_{1}\right)} \sum_{k=0}^{\infty} \frac{a^{k}\left(\frac{1-\beta+\epsilon}{2}\right)_{k}}{2^{k}\left(\frac{3+2 \epsilon_{1}}{4}\right)_{k}\left(\frac{5+2 \epsilon_{1}}{4}\right)_{k}} t^{2 k+\epsilon_{1}+1 / 2} H(t) .
\end{aligned}
$$

By using the relation $G_{w, 0}(t, \tau)={ }_{R} D_{t}^{\epsilon_{1}} G_{w, \epsilon_{1}}(t, \tau)$ given in Lemma 34 to (126), or by replacing $\epsilon_{1}$ by 0 in (126), we obtain the expression for $G_{w, 0}(t, 0)=w_{2,0}(t) H(t)$ given in Lemma 33. 


\subsection{Solution of Equations (75) and (74) Satisfying Condition 2 (iii) by Frobenius' Method}

We now give the solutions of (75) and (74) by using Frobenius' method. We note that (75) is obtained from Equation (108) by replacing $\frac{d}{d t} G_{v, \epsilon_{1}}(t, \tau), \epsilon_{1}$ and $\tau$ by $y(t),-\beta+\epsilon$ and 0 , respectively. In place of (121), we use the following:

$$
y(t)=\frac{d}{d t} G_{v, 0}(t, 0)=\sum_{k=0}^{\infty} p_{k} \frac{1}{\Gamma(\alpha+2 k)} t^{\alpha+2 k-1} H(t),
$$

in Equation (75). Then we obtain (122), with $\epsilon_{1}$ replaced by $-\beta+\epsilon$. From it, we have $\alpha=1+\epsilon_{1}=1-\beta+\epsilon$, and $p_{k}$ given by (123). By using these in (127), we obtain the following:

$$
y(t)=\sum_{k=0}^{\infty} \frac{(2 a)^{k}\left(\frac{1-\beta+\epsilon}{2}\right)_{k}}{\Gamma(2 k-\beta+\epsilon+1)} t^{2 k-\beta+\epsilon} H(t)
$$

We note that (74) is obtained from Equation (108) by replacing ${ }_{R} D_{t}^{1 / 2} G_{v, \epsilon_{1}}(t, \tau), \epsilon_{1}$ and $\tau$ by $u(t),-\beta+\epsilon$ and 0 , respectively. In place of (121), we use the following:

$$
u(t)={ }_{R} D_{t}^{1 / 2} G_{v, 0}(t, 0)=\sum_{k=0}^{\infty} p_{k} \frac{1}{\Gamma\left(\alpha+2 k+\frac{1}{2}\right)} t^{\alpha+2 k-1 / 2} H(t),
$$

in Equation (75). Then, we obtain (122), with $\epsilon_{1}$ replaced by $-\beta+\epsilon$. From it, we have $\alpha=1+\epsilon_{1}=1-\beta+\epsilon$, and $p_{k}$ given by (123). By using these in (129), we obtain the following:

$$
u(t)=\sum_{k=0}^{\infty} \frac{(2 a)^{k}\left(\frac{1-\beta+\epsilon}{2}\right)_{k}}{\Gamma\left(2 k-\beta+\epsilon+\frac{3}{2}\right)} t^{2 k-\beta+\epsilon+1 / 2} H(t) .
$$

\subsection{Solutions of Equations (75) and (74) with the Aid of Theorem 3}

When Condition 2 (iii) is satisfied, Equations (21) and (20) are expressed by (75) and (74), respectively. It is stated in Lemmas 32 and 34 that particular solutions of them are obtained in terms of the Green's functions $G_{v, \epsilon_{1}}(t, 0)$ and $G_{w, \epsilon_{1}}(t, 0)$, which are given in Lemmas 31 and 33 .

By using $G_{v, \epsilon_{1}}(t, 0)$ given in (124) and Formulas (52) and (50), we obtain the following:

$$
\begin{aligned}
y_{f}(t) & ={ }_{R} D_{t}^{\beta-\epsilon+\epsilon_{1}+1} G_{v, \epsilon_{1}}(t, 0)={ }_{R} D_{t}^{\beta-\epsilon+\epsilon_{1}+1} \sum_{k=0}^{\infty} \frac{\left(\frac{1-\beta+\epsilon}{2}\right){ }_{k} 2^{k} a^{k}}{\Gamma\left(2 k+2+\epsilon_{1}\right)} t^{2 k+1+\epsilon_{1}} H(t) \\
& =\sum_{k=0}^{\infty} \frac{\left(\frac{1-\beta+\epsilon}{2}\right)_{k}(2 a)^{k}}{\Gamma(2 k+1-\beta+\epsilon)} t^{2 k-\beta+\epsilon} H(t) \\
& =\frac{1}{\Gamma(1-\beta+\epsilon)} \sum_{k=0}^{\infty} \frac{a^{k}}{2^{k}\left(\frac{-\beta+\epsilon}{2}+1\right)_{k}} t^{2 k-\beta+\epsilon} H(t) .
\end{aligned}
$$

This solution agrees with $y(t)$ given by (93) and (128), which are obtained by solving Equation (75).

Lemma 35. When $\beta=2 n+1$ for $n \in \mathbb{Z}_{>-1}$, (131) is expressed by the following:

$$
y_{f}(t)=\epsilon(2 n) ! \sum_{k=0}^{\infty} \frac{a^{k}}{\left(\frac{1}{2}-n\right)_{k} 2^{k}} t^{2 k-2 n-1+\epsilon} H(t) .
$$


When $\beta=2 n$ for $n \in \mathbb{Z}_{>0}$, (131) is expressed by the following:

$$
\begin{aligned}
y_{f}(t)= & -\epsilon \sum_{k=0}^{n-1}(2 a)^{k}\left(\frac{1}{2}-n\right)_{k}(2 n-2 k-1) ! t^{2 k-2 n+\epsilon} H(t) \\
& +\sum_{k=n}^{\infty} \frac{(2 a)^{k}\left(\frac{1}{2}-n\right)_{k}}{(2 k-2 n) !} t^{2 k-2 n+\epsilon} H(t)
\end{aligned}
$$

Proof. By putting $\beta=2 n+1$ in the last member of Equation (131) and using the following formula:

$$
\frac{1}{\Gamma(z)}=\frac{\sin (\pi z) \Gamma(1-z)}{\pi}
$$

we obtain (132). By putting $\beta=2 n$ in the fourth member of Equation (131) and using the formula (134), we obtain (133).

By using $G_{w, \epsilon_{1}}(t, 0)$ given in (126) and Formula (51), we obtain the following:

$$
\begin{aligned}
u_{f}(t) & ={ }_{R} D_{t}^{\beta-\epsilon+\epsilon_{1}} G_{w, \epsilon_{1}}(t, 0)={ }_{R} D_{t}^{\beta-\epsilon+\epsilon_{1}} \sum_{k=0}^{\infty} \frac{\left(\frac{1-\beta+\epsilon}{2}\right)_{k} 2^{k} a^{k}}{\Gamma\left(2 k+\epsilon_{1}+\frac{3}{2}\right)} t^{2 k+\epsilon_{1}+1 / 2} \\
& =\sum_{k=0}^{\infty} \frac{\left(\frac{1-\beta+\epsilon}{2}\right)_{k}(2 a)^{k}}{\Gamma\left(2 k+\frac{3}{2}-\beta+\epsilon\right)} t^{2 k-\beta+\epsilon+1 / 2} H(t) \\
& =\frac{1}{\Gamma\left(\frac{3}{2}-\beta+\epsilon\right)} \sum_{k=0}^{\infty} \frac{a^{k}\left(\frac{1-\beta+\epsilon}{2}\right)_{k}}{2^{k}\left(\frac{3-2 \beta+2 \epsilon}{4}\right)_{k}\left(\frac{5-2 \beta+2 \epsilon}{4}\right)_{k}} t^{2 k-\beta+1 / 2+\epsilon} H(t) .
\end{aligned}
$$

This solution agrees with $u(t)$ given by (95), which is obtained by solving Equation (74). Lemma 36. When $\beta=n+\frac{3}{2}$ for $n \in \mathbb{Z}_{>-1}$, (135) is expressed by the following:

$$
\begin{aligned}
u_{f}(t)= & \epsilon(-1)^{n} \sum_{k=0}^{\left\lfloor\frac{n}{2}\right\rfloor}(n-2 k) !\left(\frac{-2 n-1}{4}\right)_{k}(2 a)^{k} t^{2 k-n-1+\epsilon} H(t) \\
& +\sum_{k=\left\lfloor\frac{n}{2}\right\rfloor+1}^{\infty} \frac{\left(\frac{-2 n-1}{4}\right)_{k}(2 a)^{k}}{(2 k-n-1) !} t^{2 k-n-1+\epsilon} H(t)
\end{aligned}
$$

where $\left\lfloor\frac{n}{2}\right\rfloor$ is the greatest integer not exceeding $\frac{n}{2}$. When $\beta=2 n+1$ for $n \in \mathbb{Z}_{>-1}$, (135) is expressed by the following:

$$
\begin{aligned}
u_{f}(t)= & n ! \sum_{k=0}^{n} \frac{(-1)^{k}(2 a)^{k}}{\Gamma\left(2 k-2 n+\frac{1}{2}+\epsilon\right)(n-k) !} t^{2 k-2 n+\epsilon-1 / 2} H(t) \\
& +\epsilon(-1)^{n} \frac{n !}{2} \sum_{k=n+1}^{\infty} \frac{(k-n-1) !(2 a)^{k}}{\Gamma\left(2 k-2 n+\frac{1}{2}+\epsilon\right)} t^{2 k-2 n+\epsilon-1 / 2} H(t) .
\end{aligned}
$$

Proof. By putting $\beta=n+\frac{3}{2}$ in the fourth member of Equation (135) and using Formula (134), we obtain (136). By using $\beta=2 n+1$, we obtain (137). 


\section{Conclusions}

In [3], the problem of obtaining the particular solution of an inhomogeneous ordinary differential equation with polynomial coefficients is discussed in terms of the Green's function, in the framework of distribution theory. In Section 2, a compact recipe is presented, which is applicable to the case of an inhomogeneous fractional differential equation, which is expressed by Equation(15). In the recipe, the particular solution is given by Theorems 1, 2 or 3 , according as the inhomogeneous part satisfies Condition 2 (i), (ii) or (iii), in the framework of nonstandard analysis.

In Sections 3-3.4, the complementary solutions and the Green's functions are given for Equation (18) and the related ordinary differential equation (19). They are used to give the particular solutions of Equations (18) and (19), with the aid of Theorem 1 in Sections 3.2 and 3.3, when Condition 2 (i) is satisfied. In Section 3.4, the Green's functions are obtained by the operational calculus or the method of iterations.

When $b=0$, Equations (18) and (19) are reduced to Equations (20) and (21), respectively. Equation (20) for $a=-1$ is the equation, which was studied by Kim and O [14]. Sections 5 and 6 are focused on the solution of Equations (20) and (21), which satisfy Conditions 2 (ii) and 2 (iii).

In Section 4, we consider a fractional differential equation (22) which satisfies Condition 2 (iii), and is solved by the operational calculus or the method of iterations. Equations (20) and (21), which satisfy Conditions 2 (iii) are denoted by Equations (74) and (75). They are special ones of (22), and their solutions by iterations are given in Sections 4.3 and 4.2, respectively, without using a transformed differential equation. The solutions with the aid of Theorems 1 and 3, are given in Sections 3.5 and 5.2-5.7, respectively. Based on Theorems 1 and 3, we obtain the same results, although the derivation in the former is restricted to the case of Condition 2 (i). The solutions by using Frobenius' method are given in Section 5.6. In Section 5.7, nonstandard solutions of Equations (74) and (75) are given in the form where infinitesimal terms appear.

The solutions of Equations (20) and (21), which satisfy Condition 2 (ii), are given with the aid of Theorem 2, in Sections 5-5.4.

Author Contributions: Author K.-i.S. showed T.M. the paper [14]. Then, T.M. wrote a draft of this paper. Afterward, both authors collaborated to complete this manuscript. Both authors have read and agreed the published version of the manuscript.

Funding: This research received no external funding.

Acknowledgments: The authors are grateful to the reviewers of this paper. Following their suggestions, the original Section 1 was divided into the present Sections 1 and 2, Remark 1 was added, and the abstract was revised.

Conflicts of Interest: Authors have declared that no competing interests exist.

\section{References}

1. Morita, T.; Sato, K. Liouville and Riemann-Liouville Fractional Derivatives via Contour Integrals. Frac. Calc. Appl. Anal. 2013, 16, 630-653. [CrossRef]

2. Podlubny, I. Fractional Differential Equations; Section 2.3.2; Academic Press: San Diego, CA, USA, 1999.

3. Morita, T.; Sato, K. Solution of Inhomogeneous Differential Equations with Polynomial Coefficients in Terms of the Green's Function. Mathematics 2017, 5, 62. [CrossRef]

4. Morita, T.; Sato, K. Solution of Inhomogeneous Differential Equations with Polynomial Coefficients in Terms of the Green's Function and AC-Laplace Transform. J. Adv. Math. Comput. Sc. 2018, 28, 1-22. [CrossRef]

5. Morita, T. Solution of Euler's Differential Equation and AC-Laplace Transform of Inverse Power Functions and Their Pseudofunctions, in Nonstandard Analysis. J. Adv. Math. Comput. Sci. 2021, 36, 47-60. [CrossRef]

6. Schwartz, L. Théorie des Distributions; Hermann: Paris, France, 1966.

7. Gelfand, I.M.; Silov, G.E. Generalized Functions. Volume 1; Academic Press Inc.: New York, NY, USA, 1964.

8. Zemanian, A.H. Distribution Theory and Transform Analysis; Dover Publ. Inc.: New York, NY, USA, 1965.

9. Diener, F.; Diener, M. Tutorial. In Nonstandard Analysis in Practice; Springer: Berlin/Heidelberg, Germany, 1995; pp. 1-21.

10. Morita, T.; Sato, K. A Study on the Solution of Linear Differential Equations with Polynomial Coefficients. J. Adv. Math. Comput. Sci. 2018, 28, 1-15. [CrossRef] 
11. Morita, T.; Sato, K. Solution of Fractional Differential Equation in Terms of Distribution Theory. Interdiscip. Inf. Sci. 2006, 12, 71-83. [CrossRef]

12. Morita, T.; Sato, K. Neumann-series Solution of Fractional Differential Equation. Interdiscip. Inf. Sci. 2016, 16, 127-137. [CrossRef]

13. Riesz, F.; Sz-Nagy, B. Functional Analysis; Dover Publ. Inc.: Mineola, NY, USA, 1990; Chapter IV.

14. Kim, M.-H.; O, H.-C. Explicit Representation of Green's Function for Linear Fractional Differential Operator with Variable Coefficients. J. Fract. Calc. Appl. 2014, 5, 26-36.

15. Magnus, M.; Oberhettinger, F. Formulas and Theorems for the Functions of Mathematical Physics; Chelsea Publ. Co.: New York, NY, USA, 1949. 